\title{
The Drug-Drug Interaction Potential of Antiviral Agents for the Treatment of Chronic Hepatitis C Infection
}

\author{
Kimberly L. Garrison, Polina German, Erik Mogalian, and Anita Mathias \\ Gilead Sciences Inc., Foster City, California
}

Received October 18, 2017; accepted March 26, 2018

\begin{abstract}
Several safe and highly effective direct-acting antiviral (DAA) drugs for chronic hepatitis $\mathrm{C}$ virus (HCV) have been developed and greatly increase the number of therapeutic options available to successfully treat $\mathrm{HCV}$ infection. However, because treatment regimens contain at least two drugs (e.g., elbasvir and grazoprevir, glecaprevir and pibrentasvir, or sofosbuvir with daclatasvir, simeprevir, ledipasvir, or velpatasvir) and up to five drugs (ombitasvir/paritaprevir/ritonavir
\end{abstract}

plus dasabuvir with or without ribavirin), the potential for drug-drug interactions (DDIs) becomes an important consideration for HCVinfected individuals with comorbidities that require concomitant medications, such as human immunodeficiency virus/HCV coinfection or immunosuppression after liver transplantation. This review details the pharmacokinetics and DDI potential of approved DAAs for the treatment of HCV infection.

\section{Introduction}

Chronic hepatitis $\mathrm{C}$ virus (HCV) is a global health problem affecting an estimated 71 million people worldwide (http://www.who.int/mediacentre/ factsheets/fs 164/en/). In the United States, an estimated 2.7-3.9 million people have chronic HCV infection, which causes more than 19,000 deaths annually http://www.euro.who.int/en/health-topics/communicable-diseases/hepatitis/data-and-statistics, although under-reporting may contribute to an underestimation by up to 5-fold (Mahajan et al., 2014). In the European Region, approximately 15 million people have chronic HCV infection, which results in 86,000 deaths annually in WHO European Member States. (http://www.who.int/mediacentre/factsheets/ fs $164 / \mathrm{en} /$ ). After acute infection with $\mathrm{HCV}$, approximately $75 \%-85 \%$ of individuals fail to spontaneously clear the virus and thus progress to chronic infection. Complications of chronic HCV infection include liver cirrhosis, end-stage liver disease, and hepatocellular carcinoma; in the United States, HCV remains one of the leading indications for liver transplantation (Kim et al., 2017). Almost three-quarters of the reported HCV-related deaths in the United States have occurred in the baby boomer generation, with a median age at death (57 years) that is approximately 20 years less than the average lifespan for the general population (Smith et al., 2012).

Prior to 2011, the standard of care for treating HCV included administration of pegylated-interferon (Peg-IFN) and ribavirin. These

\footnotetext{
The work was supported by Gilead Sciences Inc. https://doi.org/10.1124/dmd.117.079038.
}

medications were associated with frequent adverse effects and inadequate antiviral efficacy (assessed as sustained virologic response). In 2013, the U.S. Food and Drug Administration approved the first directacting antiviral (DAA) protease inhibitors, boceprevir and telaprevir. When administered with Peg-IFN plus ribavirin, boceprevir- and telaprevir-containing regimens resulted in higher sustained virologic response rates compared with Peg-IFN plus ribavirin monotherapy, but the safety and efficacy of these regimens remained suboptimal. Since then, many highly effective and well tolerated DAA regimens have been developed, consisting of various combinations of nucleotide analog NS5B inhibitors, non-nucleotide NS5B inhibitors, NS5A inhibitors, and/or NS3/4A protease inhibitors, including simeprevir, daclatasvir, sofosbuvir, ledipasvir/sofosbuvir, ombitasvir/paritaprevir/ritonavir plus dasabuvir, sofosbuvir/velpatasvir, elbasvir/grazoprevir, sofosbuvir/ velpatasvir/voxilaprevir, and glecaprevir/pibrentasvir. The combination therapies now achieve cure rates approaching $100 \%$, and regimens have even become available for those patients who fail the available, highly effective DAA regimens (Vosevi, 2017a,b).

Given the significant and rapid advancements in highly effective treatment options, boceprevir and telaprevir (which are no longer available commercially) and asunaprevir (an NS3 protease inhibitor, which is only approved for use in China and Japan) will not be further discussed in this article.

The availability of highly effective and well tolerated DAA regimens represents a significant advancement for patients with chronic HCV infection; however, the potential for metabolic or transport-mediated

ABBREVIATIONS: 3TC, lamivudine; ABC, abacavir; ALT, alanine aminotransferase; ARV, antiretroviral; ATV, atazanavir; AUC, area under the plasma concentration versus time curve; BCRP, breast cancer resistance protein; COBI, cobicistat; DAA, direct-acting antiviral; DDI, drug-drug interaction; DRV, darunavir; DTG, dolutegravir; EE, ethinyl estradiol; EFV, efavirenz; EVG, elvitegravir; FTC, emtricitabine; GS-331007, 1-((2R,3R,4R,5R)-3-fluoro-4-hydroxy-5-(hydroxymethyl)-3-methyl-tetrahydrofuran-2-yl)pyrimidine-2,4-dione; HCV, hepatitis C virus; HIV, human immunodeficiency virus; LPV, lopinavir; MRP, multidrug resistance protein; NRTI, nucleoside or nucleotide reverse transcriptase inhibitor; OATP, organic anion-transporting polypeptide; P-gp, p-glycoprotein; P450, cytochrome P450; Peg-IFN, pegylated-interferon; RAL, raltegravir; RPV, rilpivirine; SmPC, European Summary of Product Characteristics; TAF, tenofovir alafenamide; TDF, tenofovir disoproxil fumarate; TFV, tenofovir; UGT, uridine glucuronosyltransferase; USPI, U.S. Prescribing Information. 
drug-drug interactions (DDIs) warrants consideration because these treatment regimens contain at least two drugs (e.g., ledipasvir/sofosbuvir) and up to five drugs (ombitasvir/paritaprevir/ritonavir plus dasabuvir plus ribavirin). Careful thought about possible DDIs is especially important for patients with chronic conditions such as human immunodeficiency virus (HIV)/HCV coinfection, individuals who receive immunosuppressive therapy after liver transplantation, or patients with other comorbidities that require concomitant medication. This review details the pharmacokinetics and DDI potential of currently approved DAAs for the treatment of $\mathrm{HCV}$ infection.

\section{Summary of DAA Characteristics}

Below is a list of DAAs available as single agents (to be used in combination with other DAAs) or as complete treatment regimens. Table 1 summarizes clinical and/or nonclinical data characterizing DAAs as substrates, inducers, or inhibitors of metabolic enzymes and transporters.

\section{Simeprevir}

Simeprevir (150 mg), an NS3/4A protease inhibitor, is administered once daily in combination with sofosbuvir or Peg-IFN plus ribavirin in subjects with HCV genotype 1 infection (Olysio, 2017). Simeprevir is dosed with food because food increases its bioavailability [area under the plasma concentration versus time curve (AUC)] by 61\%-69\% (Olysio, 2016, 2017). Simeprevir is primarily eliminated in the feces $(91 \%)$ predominantly as metabolites, with $<1 \%$ excreted in the urine. Simeprevir is a substrate of CYP3A, P-glycoprotein (P-gp)/breast cancer resistance protein $(\mathrm{BCRP})$, multidrug resistance protein $\mathrm{MRP} 2$, and organic anion-transporting polypeptide OATP1B1/3 and inhibits CYP1A2, CYP3A4 (intestinal), P-gp, and OATP1B1/3 (OuwerkerkMahadevan et al., 2016; Olysio, 2017).

\section{Sofosbuvir}

Sofosbuvir, a nucleotide analog prodrug, is converted to the active triphosphate that directly inhibits HCV NS5B RNA polymerase. Sofosbuvir (400 mg) exhibits pangenotypic potency and is indicated for use with other DAAs, including simeprevir, daclatasvir, ledipasvir, or velpatasvir (Daklinza, 2017; Epclusa, 2017b; Harvoni, 2017b; Sovaldi, 2017a). Sofosbuvir is recommended for administration once daily without regard to food in the U.S. Prescribing Information (USPI) and with food in the European Summary of Product Characteristics (SmPC) (Sovaldi, 2017a,b). Sofosbuvir is extensively metabolized by hydrolases, and the GS-331007 metabolite accounts for $>90 \%$ of overall drug exposure (Kirby et al., 2015). Sofosbuvir (but not GS-331007) is a substrate of P-gp/BCRP. Neither sofosbuvir nor its metabolites inhibit or induce metabolizing enzymes or drug transporters.

\section{Daclatasvir}

Daclatasvir $(60 \mathrm{mg})$ is an NS5A inhibitor approved for use with asunaprevir for HCV genotype 1 (China and Japan) or with sofosbuvir for $\mathrm{HCV}$ genotypes 1, 3, or 4 (United States and European Union) (Daklinza, 2014, 2017). Daclatasvir is administered once daily without regard to food. The primary route of elimination for daclatasvir is the feces $(88 \%)$, predominantly as the parent, with little renal excretion (6.6\%). Daclatasvir is a substrate for CYP3A and P-gp and is an inhibitor of P-gp/BCRP and OAT1B1/3 (Garimella et al., 2016; Daklinza, 2017).

\section{Ledipasvir/Sofosbuvir}

A fixed-dose combination of NS5A inhibitor ledipasvir $(90 \mathrm{mg}$ ) and sofosbuvir (400 mg; presented above) is approved for treatment of $\mathrm{HCV}$ genotype 1, 4, 5, or 6 infection. Ledipasvir/sofosbuvir is administered once daily without regard to food (Harvoni, 2017a,b). Ledipasvir is primarily excreted unchanged in bile, with $<1 \%$ excreted in urine (Kirby et al., 2013). Ledipasvir is both a substrate and an inhibitor of P-gp/BCRP; ledipasvir does not inhibit or induce cytochrome P450 (P450) enzymes or uridine glucuronosyltransferases (UGTs) (German et al., 2014b).

\section{Ombitasvir/Paritaprevir/Ritonavir With or Without Dasabuvir}

The combination of ombitasvir (25 mg), an NS5A inhibitor, with a ritonavir (100 mg)-boosted NS3/4A protease inhibitor, paritaprevir (150 mg), plus a non-nucleotide NS5B inhibitor, dasabuvir (150 mg twice daily), with or without ribavirin (with food) is approved for treatment of HCV genotype 1 infection (Viekira Pak, 2017; Viekirax, 2018). Ombitasvir/paritaprevir/ritonavir is also approved for use with ribavirin against HCV genotype 4 (Technivie, 2016; Viekirax, 2018). Ombitasvir, ritonavir, paritaprevir, and dasabuvir are primarily eliminated in the feces, whereas $1.91 \%, 8.8 \%, 11.3 \%$, and approximately $2 \%$ of the doses are excreted in the urine, respectively (Viekira Pak, 2017; Viekirax, 2018). Ombitasvir is primarily excreted unchanged, whereas paritaprevir, ritonavir, and dasabuvir are primarily metabolized. Ombitasvir is a substrate of amidases, CYP3A, and P-gp/BCRP. Paritaprevir is a substrate for CYP3A, P-gp/BCRP, and OATP1B1/3. Dasabuvir is a substrate for CYP2C8 and P-gp/BCRP (Menon et al., 2015). Ombitasvir, paritaprevir, and dasabuvir all inhibit UGT1A1 (Viekira Pak, 2017). In addition, paritaprevir inhibits BCRP and OATP1B1/3, and dasabuvir inhibits BCRP (Viekira Pak, 2017). To increase its systemic exposure, paritaprevir is coadministered with low-dose ritonavir (Norvir, 2017).

\section{Sofosbuvir/Velpatasvir}

Velpatasvir (100 mg) is a pangenotypic HCV NS5A inhibitor administered as a fixed-dose combination with sofosbuvir (400 mg; presented above) that is approved for treatment of HCV genotypes 1, 2, 3, 4, 5, or 6 and can be taken without regard to food (Epclusa, 2017a,b). Velpatasvir is primarily eliminated unchanged in the feces, with $<1 \%$ excreted renally (Mogalian et al., 2015b). Velpatasvir is a substrate of P-gp/BCRP, CYP2B6, CYP2C8, and CYP3A4 and inhibits P-gp, BCRP, and OATP (Mogalian et al., 2016b). Velpatasvir does not induce or inhibit P450s or UGTs (Mogalian et al., 2016b).

\section{Elbasvir/Grazoprevir}

Elbasvir (50 mg), an NS5A inhibitor, and grazoprevir (100 mg), an NS3/4A protease inhibitor, are approved for treatment of $\mathrm{HCV}$ genotype 1 or 4 infection and can be taken without regard to food (Zepatier, 2017, 2018). Elbasvir and grazoprevir are primarily eliminated in the feces, with $<1 \%$ in the urine; both are subject to oxidative metabolism, primarily by CYP3A (Zepatier, 2017, 2018). Elbasvir and grazoprevir are substrates of CYP3A and P-gp; the role of P-gp in the absorption of both drugs appears to be minimal (Zepatier, 2017, 2018). Grazoprevir may also be transported by BCRP (Zepatier, 2018). Grazoprevir is a substrate of OATP1B1/3 (Zepatier, 2017, 2018). Elbasvir is not a CYP3A inhibitor in vitro and grazoprevir is a weak CYP3A inhibitor in humans (Zepatier, 2017). In vitro, elbasvir inhibits P-gp and both drugs inhibit BCRP (Zepatier, 2017, 2018).

\section{Sofosbuvir/Velpatasvir/Voxilaprevir}

The combination sofosbuvir/velpatasvir/voxilaprevir is approved for treatment of HCV genotypes 1, 2, 3, 4, 5, or 6, including for patients who have failed other DAA regimens, and is recommended to be administered with food (Vosevi, 2017a,b). The characteristics of sofosbuvir and 
TABLE 1

Summary of potential DDI mechanisms

\begin{tabular}{|c|c|c|c|c|c|c|c|c|c|c|c|c|c|c|}
\hline \multirow{3}{*}{$\begin{array}{l}\text { Enzyme or } \\
\text { Transporter }\end{array}$} & \multicolumn{14}{|c|}{ HCV DAA } \\
\hline & \multirow{2}{*}{ SMP } & \multirow{2}{*}{ SOF } & \multirow{2}{*}{$\begin{array}{c}\mathrm{DCV}+ \\
\mathrm{SOF}\end{array}$} & \multirow{2}{*}{$\frac{\mathrm{LDV} / \mathrm{SOF}}{\mathrm{LDV}}$} & \multicolumn{4}{|c|}{$\mathrm{OBV} / \mathrm{PTV} / \mathrm{r} \pm \mathrm{DSV}$} & \multicolumn{2}{|c|}{ EBR/GZR } & \multirow{2}{*}{$\frac{\text { SOF/VEL }}{\text { VEL }}$} & \multirow{2}{*}{$\frac{\text { SOF/VEL/VOX }}{\text { VOX }}$} & \multicolumn{2}{|c|}{ GLE/PIB } \\
\hline & & & & & OBV & PTV & RTV & DSV & EBR & GZR & & & GLE & PIB \\
\hline BCRP & SUB, INH & SUB & INH & $\begin{array}{l}\text { SUB, } \\
\text { INH }^{a}\end{array}$ & SUB & $\begin{array}{l}\text { SUB, } \\
\text { INH }\end{array}$ & INH & $\begin{array}{l}\text { SUB, } \\
\text { INH }\end{array}$ & $\mathrm{INH}^{a}$ & $\mathrm{INH}^{a}$ & $\begin{array}{l}\text { SUB, } \\
\text { INH }^{a}\end{array}$ & $\mathrm{SUB}, \mathrm{INH}^{a}$ & $\begin{array}{l}\text { SUB, } \\
\text { INH }\end{array}$ & $\begin{array}{c}\text { SUB, } \\
\text { INH }\end{array}$ \\
\hline OATP1B1/1B3 & SUB, INH & - & INH & - & - & $\begin{array}{l}\text { SUB, } \\
\text { INH }\end{array}$ & - & - & - & SUB & $\begin{array}{l}\text { SUB, } \\
\text { INH }^{a}\end{array}$ & SUB, $\mathrm{INH}^{a}$ & $\begin{array}{l}\text { SUB, } \\
\text { INH }\end{array}$ & INH \\
\hline OATP2B1 & SUB & - & - & - & - & INH & INH & - & - & - & $\mathrm{INH}^{a}$ & - & - & - \\
\hline P-gp & SUB, INH & SUB & $\begin{array}{l}\text { SUB, } \\
\text { INH }\end{array}$ & $\begin{array}{l}\text { SUB, } \\
\text { INH }^{a}\end{array}$ & SUB & SUB & $\begin{array}{l}\text { SUB, } \\
\text { INH }\end{array}$ & SUB & $\begin{array}{l}\text { SUB, } \\
\text { INH }\end{array}$ & SUB & $\begin{array}{l}\text { SUB, } \\
\text { INH }^{a}\end{array}$ & $\mathrm{SUB}, \mathrm{INH}^{a}$ & $\begin{array}{l}\text { SUB, } \\
\text { INH }\end{array}$ & $\begin{array}{c}\text { SUB, } \\
\text { INH }\end{array}$ \\
\hline CYP1A2 & INH (weak) & - & - & - & $c$ & - & - & - & - & - & - & SUB & INH & INH \\
\hline CYP2B6 & - & - & - & - & - & - & - & - & - & - & SUB & - & - & - \\
\hline CYP2C8 & - & - & - & - & - & - & - & SUB & - & - & SUB & SUB & - & - \\
\hline CYP3A4 & $\begin{array}{l}\mathrm{SUB}, \mathrm{INH} \\
(\text { weak) }\end{array}$ & - & $\begin{array}{l}\text { SUB, } \\
\text { IND }^{b}\end{array}$ & - & SUB & SUB & $\begin{array}{l}\text { SUB, } \\
\text { INH }\end{array}$ & - & SUB & $\begin{array}{l}\text { SUB, INH } \\
\text { (weak) }\end{array}$ & SUB & SUB & $\begin{array}{l}\mathrm{SUB}^{d} \\
\text { INH }\end{array}$ & INH \\
\hline UGT1A1 & - & - & - & - & INH & INH & - & INH & - & - & - & - & INH & INH \\
\hline
\end{tabular}

DCV, daclatasvir; DSV, dasabuvir; EBR, elbasvir; GLE, glecaprevir; GZR, grazoprevir; IND, inducer; INH, inhibitor; LDV, ledipasvir; OBV, ombitasvir; PIB, pibrentasvir; PTV, paritaprevir; RTV or/r, ritonavir; SMP, simeprevir; SOF, sofosbuvir; SUB, substrate; VEL, velpatasvir; VOX, voxilaprevir.

${ }^{a}$ Limited primarily to the process of absorption/intestine (Epclusa, 2017; Harvoni, 2017; Zepatier, 2018).

${ }^{b}$ Very weak inducer in vivo with no dose adjustment of substrates needed (Daklinza, 2018).

${ }^{c}$ CYP1A2 substrates may require monitoring (Viekirax, 2018).

velpatasvir are presented above. Voxilaprevir is a pangenotypic NS3/4A inhibitor that is exclusively eliminated in the feces, with slow metabolic turnover by predominantly CYP3A (Kirby et al., 2016b; Vosevi, 2017a,b). Voxilaprevir is a substrate and inhibitor of P-gp, BCRP, and OATP1B1/3 (Kirby et al., 2016a; Vosevi, 2017a,b).

\section{Glecaprevir/Pibrentasvir}

The combination of NS3/4A inhibitor glecaprevir (100 mg) and NS5A inhibitor pibrentasvir $(40 \mathrm{mg})$ is approved for treatment of $\mathrm{HCV}$ genotype $1,2,3,4,5$, or 6 infection, including patients with genotype 1 who have failed either an NS5A- or NS3/4a-containing regimen. Biliary excretion is the primary route of elimination for both glecaprevir and pibrentasvir, with $0.7 \%$ and $0 \%$ excreted in the urine, respectively. Glecaprevir is a substrate of P-gp, BCRP, and OATP1B1/3. Pibrentasvir is a substrate of P-gp, and transport by BCRP could not be excluded. Both glecaprevir and pibrentasvir are inhibitors of P-gp, BCRP, and OATP1B1/3, as well as weak inhibitors of CYP3A, CYP1A2, and UGT1A1.

\section{DDIs}

The potential for DDIs has been assessed mechanistically using established phenotypic probes and was also evaluated clinically using concomitant medications that may be commonly administered to HCVinfected patients. These DDI mechanisms and a selection of common concomitant medications are highlighted in Tables 2 and 3. Information pertaining to DDIs between DAAs and HIV antiretroviral (ARV) drugs is presented in Tables 4 and 5 .

\section{Mechanistic DDIs}

DAAs as Victims of Metabolic and/or Transport Inducers. Several P450s and drug transporters are involved, to varying extents, in the disposition of DAAs described above. Inducers of P450 and drug transporters, such as P-gp, may significantly decrease plasma concentrations, thereby leading to a reduced therapeutic effect. Clinical studies have been conducted to assess the sensitivity of several DAAs to metabolic and/or transporter induction using prototypical inducers (rifampin or carbamazepine).
The AUC and the plasma concentration at the end of the dosing interval $(C \tau)$ of simeprevir, a substrate for CYP3A and P-gp, were $48 \%$ and $92 \%$ lower, respectively, when coadministered with multiple-dose rifampin (600 mg) (Olysio, 2017). An increase in the simeprevir $C_{\max }$ of $31 \%$ was observed and attributed to rifampin-mediated OATP inhibition (Ouwerkerk-Mahadevan et al., 2016). Accordingly, the use of simeprevir with moderate to strong CYP3A/P-gp inducers is not recommended (Olysio, 2017).

Sofosbuvir, ledipasvir, velpatasvir, daclatasvir, voxilaprevir, glecaprevir, and pibrentasvir are P-gp substrates. Velpatasvir (minor), voxilaprevir (minor), and daclatasvir are also substrates for CYP3A. Consistent with the effect of intestinal efflux transporters on sofosbuvir pharmacokinetics, rifampin significantly reduced the sofosbuvir AUC (72\% lower) and $C_{\max }$ (77\% lower); AUC and $C_{\max }$ values were unaltered for GS-331007 (Garrison et al., 2014). Rifampin administration decreased systemic exposures of NS5A inhibitors ledipasvir, velpatasvir, daclatasvir, and pibrentasvir. AUC and $C_{\max }$ values were $59 \%$ and $35 \%$ lower for ledipasvir (German et al., 2014b), $82 \%$ and $71 \%$ lower for velpatasvir (Mogalian et al., 2016b), 79\% and 66\% lower for daclatasvir (Daklinza, 2017), and 87\% and 83\% lower for pibrentasvir (Mavyret, 2017), respectively. Systemic exposures of NS3/4A inhibitors were also decreased with multiple-dose rifampin. The AUC for voxilaprevir was $73 \%$ lower, whereas the $C_{\max }$ remained unchanged (Garrison et al., 2014; Kirby et al., 2016a). AUC and $C_{\max }$ values were $88 \%$ and $86 \%$ lower for glecaprevir, respectively (Mavyret, 2017). The use of rifampin with sofosbuvir, ledipasvir/sofosbuvir, or sofosbuvir/ velpatasvir is not recommended (Epclusa, 2017b; Harvoni, 2017b; Sovaldi, 2017b). Use of rifampin with sofosbuvir/velpatasvir/voxilaprevir or glecaprevir/pibrentasvir is contraindicated (Mavyret, 2017; Vosevi, 2017a). Coadministration of daclatasvir with strong CYP3A inducers, such as rifampin, is contraindicated (Daklinza, 2017). Use of efavirenz (EFV), a moderate inducer, is not recommended for sofosbuvir/velpatasvir, sofosbuvir/velpatasvir/voxilaprevir, and glecaprevir/pibrentasvir; no dose adjustment is needed for ledipasvir/sofosbuvir or sofosbuvir (Epclusa, 2017b; Harvoni, 2017b; Mavyret, 2017; Vosevi, 2017a). Coadministration of daclatasvir with moderate CYP3A inducers (e.g., bosentan, dexamethasone; see label) warrants a dose increase to $90 \mathrm{mg}$ daclatasvir (Daklinza, 2017). 
TABLE 2

Impact of coadministered drugs on DAA exposure (AUC)

Data are given in percentages.

\begin{tabular}{|c|c|c|c|c|c|c|c|c|c|c|c|c|c|c|}
\hline \multirow{2}{*}{ Drug Class } & \multirow{2}{*}{ SMP } & \multirow{2}{*}{ SOF } & \multirow{2}{*}{$\frac{\mathrm{LDV} / \mathrm{SOF}}{\mathrm{LDV}}$} & \multirow{2}{*}{ DCV } & \multicolumn{4}{|c|}{ OBV/PTV/DSV/r } & \multicolumn{2}{|c|}{ EBR/GZR } & \multirow{2}{*}{$\frac{\text { SOF/VEL }}{\text { VEL }}$} & \multirow{2}{*}{$\frac{\text { SOF/VEL/VOX }}{\text { VOX }}$} & \multicolumn{2}{|c|}{ GLE/PIB } \\
\hline & & & & & OBV & PTV & DSV & $/ \mathrm{r}$ & GZR & EBR & & & GLE & PIB \\
\hline \multicolumn{15}{|l|}{ Immunosuppressants } \\
\hline Cyclosporine & $\uparrow 468^{a}$ & $\uparrow 353^{b}$ & - & $\uparrow 40^{d}$ & $\leftrightarrow$ & $\uparrow 72$ & $\uparrow 30$ & $\leftrightarrow$ & $\uparrow 1421^{c, d}$ & $\uparrow 98$ & $\uparrow 103^{b}$ & $\uparrow 839^{b}$ & $\uparrow 408^{c d}$, & $\uparrow 93^{c}$ \\
\hline Everolimus & - & - & - & - & $\leftrightarrow$ & $\uparrow 26$ & $\leftrightarrow$ & $\leftrightarrow$ & & & - & - & - & - \\
\hline Mycophenolate mofetil & - & - & - & - & - & - & - & - & $\downarrow 26$ & $\leftrightarrow$ & - & - & - & - \\
\hline Sirolimus & - & - & - & - & $\leftrightarrow$ & $\uparrow 19$ & $\leftrightarrow$ & $\leftrightarrow$ & & & - & - & - & - \\
\hline Tacrolimus & $\uparrow 90^{a}$ & $\uparrow 13$ & - & - & & $\downarrow 34$ & $\leftrightarrow$ & $\leftrightarrow$ & $\leftrightarrow$ & $\leftrightarrow$ & - & - & - & - \\
\hline \multicolumn{15}{|l|}{ Statins and lipid-lowering agents } \\
\hline Atorvastatin & - & - & - & - & - & - & - & - & $\uparrow 26$ & - & - & - & - & - \\
\hline Gemfibrozil & - & - & - & - & - & $\uparrow 38$ & $\uparrow 1025$ & $\leftrightarrow$ & - & - & - & - & - & - \\
\hline Lovastatin & - & - & - & - & - & - & - & - & - & - & - & - & - & - \\
\hline Pitavastatin & - & - & - & - & - & - & - & - & $\leftrightarrow$ & - & - & - & - & - \\
\hline Pravastatin & - & - & - & - & $\leftrightarrow$ & $\leftrightarrow$ & $\leftrightarrow$ & & $\uparrow 24$ & $\leftrightarrow$ & - & - & - & - \\
\hline Rosuvastatin & - & - & - & - & $\leftrightarrow$ & $\uparrow 52$ & $\leftrightarrow$ & $\leftrightarrow$ & $\leftrightarrow$ & $\leftrightarrow$ & - & - & - & - \\
\hline Simvastatin & - & - & - & - & - & - & - & - & - & - & - & - & - & - \\
\hline \multicolumn{15}{|l|}{ Cardiovascular } \\
\hline Amiodarone & - & - & - & - & - & - & - & - & - & - & - & - & - & - \\
\hline Amlodipine & - & - & - & - & $\leftrightarrow$ & $\downarrow 22$ & $\leftrightarrow$ & $\leftrightarrow$ & - & - & - & - & - & - \\
\hline Digoxin & - & - & - & - & $\leftrightarrow$ & - & $\leftrightarrow$ & - & - & - & - & - & - & - \\
\hline \multicolumn{15}{|l|}{ Antifungals } \\
\hline Ketoconazole & - & - & - & $\uparrow 200$ & $\leftrightarrow$ & $\uparrow 98$ & $\uparrow 42$ & $\uparrow 57$ & $\uparrow 202$ & $\uparrow 80$ & $\uparrow 71$ & - & - & - \\
\hline Voriconazole & - & - & - & - & - & - & - & - & - & - & - & $\uparrow 84$ & - & - \\
\hline \multicolumn{15}{|l|}{ Acid-reducing agents } \\
\hline Famotidine & - & $\leftrightarrow$ & $\leftrightarrow$ & $\leftrightarrow$ & - & - & - & - & $\leftrightarrow$ & $\leftrightarrow$ & $\leftrightarrow$ & - & - & - \\
\hline Omeprazole & - & $\leftrightarrow$ & $\downarrow 4-42^{c}$ & $\leftrightarrow$ & $\leftrightarrow$ & $\leftrightarrow$ & $\leftrightarrow$ & $\leftrightarrow$ & - & - & $\downarrow 26-55^{c}$ & $\leftrightarrow$ & $\downarrow 29-51^{c}$ & $\leftrightarrow$ \\
\hline Pantoprazole & - & - & - & - & - & - & - & - & $\leftrightarrow$ & $\leftrightarrow$ & - & - & - & - \\
\hline \multicolumn{15}{|l|}{ Antibacterial agents } \\
\hline Erythromycin & $\uparrow 647$ & - & - & - & - & - & - & - & - & - & - & - & - & - \\
\hline Rifampin & $\downarrow 48$ & $\downarrow 72$ & $\downarrow 59$ & $\downarrow 79$ & - & - & - & - & $\begin{array}{c}\leftrightarrow,^{f} \\
\uparrow 921,^{g} \\
\uparrow 735^{h}\end{array}$ & $\leftrightarrow^{g, h}$ & $\begin{array}{l}\uparrow 46 \mathrm{SD}, \\
\downarrow 82 \mathrm{MD}\end{array}$ & $\begin{array}{c}\uparrow 691 \mathrm{SD}, \downarrow 73 \\
\mathrm{MD}\end{array}$ & $\begin{array}{c}\uparrow 755 \mathrm{SD}, \\
\downarrow 88 \mathrm{MD}\end{array}$ & $\begin{array}{c}\leftrightarrow \mathrm{SD}, \\
\downarrow 87 \mathrm{MD}\end{array}$ \\
\hline \multicolumn{15}{|l|}{ CNS agents } \\
\hline Buprenorphine/naloxone & - & - & - & - & - & - & - & - & $\downarrow 14$ & $\uparrow 22$ & - & - & - & - \\
\hline Carbamazepine & - & $\downarrow 48$ & - & - & $\downarrow 31$ & $\downarrow 70$ & $\downarrow 70$ & $\downarrow 87$ & - & - & - & - & $\downarrow 66$ & $\downarrow 51$ \\
\hline Escitalopram & $\downarrow 25$ & - & - & $\leftrightarrow$ & - & - & - & - & - & - & - & - & - & - \\
\hline Methadone & - & $\uparrow 30$ & - & - & - & - & - & & $\leftrightarrow$ & $\uparrow 20$ & - & - & - & - \\
\hline \multicolumn{15}{|l|}{ Other } \\
\hline $\begin{array}{l}\text { OC (norgestimate, norgestrel, } \\
\text { EE, or levonorgestrel) }\end{array}$ & - & $\leftrightarrow$ & $\leftrightarrow$ & - & $\leftrightarrow^{e}$ & $\downarrow 34^{e}$ & $\downarrow 52^{e}$ & $\downarrow 29^{e}$ & $\leftrightarrow$ & - & - & - & - & - \\
\hline
\end{tabular}

CNS, central nervous system; DCV, daclatasvir; DSV, dasabuvir; EBR, elbasvir; EE, ethinyl estradiol; GLE, glecaprevir; GZR, grazoprevir; LDV, ledipasvir; OBV, ombitasvir; OC, oral

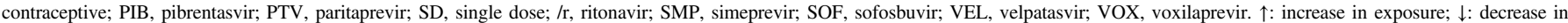
exposure; $\leftrightarrow$ : no change; —: data not available.

${ }^{a}$ Individualized dosing.

${ }^{b} 600 \mathrm{mg}$ cyclosporine.

${ }^{c}$ Depends on staggering of acid-reducing agent.

${ }^{d} 400 \mathrm{mg}$ cyclosporine.

${ }^{e} \mathrm{EE} /$ norgestimate.

${ }^{f} 600 \mathrm{mg}$ rifampin orally daily.

${ }^{8} 600 \mathrm{mg}$ rifampin intravenously SD

${ }^{h} 600 \mathrm{mg}$ rifampin orally SD.

Carbamazepine was used to assess the sensitivity of ombitasvir/ paritaprevir/ritonavir plus dasabuvir to induction. Coadministration resulted in lower exposures for all agents (AUC and $C_{\max }$ values were $30 \%$ and $31 \%$ lower for ombitasvir, $70 \%$ and $66 \%$ lower for paritaprevir, $87 \%$ and $83 \%$ lower for ritonavir, and $70 \%$ and $55 \%$ lower for dasabuvir, respectively) (Menon et al., 2015). Since marked reductions in exposure may result in a loss of antiviral efficacy, the use of strong or moderate CYP3A inducers with ombitasvir/paritaprevir/ritonavir plus dasabuvir is contraindicated (Viekira Pak, 2017).

Coadministration of elbasvir/grazoprevir, both CYP3A substrates, with strong CYP3A inducers is contraindicated based on the expectation that they will significantly decrease elbasvir and grazoprevir plasma concentrations, leading to a reduced therapeutic effect (Viekirax, 2018; Zepatier, 2018). In the absence of definitive clinical data, coadministration of elbasvir/grazoprevir with moderate CYP3A inducers is not recommended.
DAAs as Victims of Metabolic and/or Transport Inhibitors. Coadministration of DAAs with inhibitors of enzymes or transporters may alter the disposition of DAAs. Several clinical studies evaluated DAAs as victims of metabolism- or transport-mediated interactions using probe inhibitors.

Coadministration of a moderate CYP3A/P-gp inhibitor erythromycin with simeprevir (a substrate and an inhibitor of CYP3A/P-gp) resulted in a clinically relevant two-way interaction. Briefly, simeprevir AUC $\tau$ and $C_{\max }$ values were $647 \%$ and $353 \%$ higher, respectively; erythromycin AUC $\tau$ and $C_{\max }$ values were $90 \%$ and $59 \%$ higher, respectively, after coadministration (Ouwerkerk-Mahadevan et al., 2016; Olysio, 2017). As such, the use of moderate or potent CYP3A inhibitors with simeprevir is not recommended (Olysio, 2017). Simeprevir is also a substrate of hepatic uptake transporter OATP1B1/3. Coadministration with inhibitors of OATP1B1/3, such as cyclosporine, may result in higher simeprevir plasma concentrations and is not recommended (Olysio, 2017). 
TABLE 3

Impact of DAA on coadministered drug exposure (AUC)

Data are given in percentages.

\begin{tabular}{|c|c|c|c|c|c|c|c|c|c|c|}
\hline Drug Class & SMP & SOF & LDV/SOF & DCV & OBV/PTV/DSV/r & GZR & EBR & SOF/VEL & SOF/VEL/VOX & GLE/PIB \\
\hline \multicolumn{11}{|l|}{ Immunosuppressants } \\
\hline Cyclosporine & $\leftrightarrow$ & $\leftrightarrow$ & $\leftrightarrow$ & $\leftrightarrow$ & $\uparrow 482$ & $\leftrightarrow$ & $\leftrightarrow$ & - & $\leftrightarrow$ & - \\
\hline Everolimus & - & - & - & - & $\uparrow 2612$ & - & - & - & - & - \\
\hline Sirolimus & - & - & - & - & $\uparrow 3699$ & - & - & - & - & - \\
\hline Tacrolimus & $\downarrow 17$ & $\leftrightarrow$ & $\leftrightarrow$ & $\leftrightarrow$ & $\uparrow 5613$ & \multicolumn{2}{|c|}{$\uparrow 43$} & $\leftrightarrow$ & - & $\uparrow 45$ \\
\hline \multicolumn{11}{|l|}{ Statins } \\
\hline Pravastatin & - & - & - & - & $\uparrow 82$ & \multicolumn{2}{|c|}{$\uparrow 33$} & $\uparrow 35$ & $\uparrow 116^{b}$ & $\uparrow 130^{c}$ \\
\hline Rosuvastatin & $\uparrow 181$ & - & - & $\uparrow 58$ & $\uparrow 159$ & \multicolumn{2}{|c|}{$\uparrow 126$} & $\uparrow 169$ & $\uparrow 639^{b}$ & $\uparrow 115^{c}$ \\
\hline Simvastatin & $\begin{array}{c}\uparrow 51 ; \\
\text { simvastatin } \\
\text { acid } \uparrow 88\end{array}$ & - & - & - & - & - & - & - & - & $\begin{array}{c}\uparrow 132 ; \\
\text { simvastatin acid } \\
\uparrow 348\end{array}$ \\
\hline \multicolumn{11}{|l|}{ Cardiovascular } \\
\hline Losartan & - & - & - & - & - & - & - & - & - & $\begin{array}{l}\uparrow 56 ; \text { losartan } \\
\text { acid } \leftrightarrow\end{array}$ \\
\hline Valsartan & - & - & - & - & - & - & - & - & - & $\uparrow 31$ \\
\hline Antifungals & & & & & & & - & & & \\
\hline Ketoconazole & - & - & - & - & $\uparrow 117$ & - & & - & - & - \\
\hline \multicolumn{11}{|l|}{$\begin{array}{l}\text { Acid-reducing } \\
\text { agents }\end{array}$} \\
\hline Omeprazole & $\uparrow 21$ & - & - & - & $\downarrow 38$ & - & - & - & - & $\downarrow 21$ \\
\hline Antibacterial agents & & & & & & & - & & & \\
\hline Erythromycin & $\uparrow 90$ & - & - & - & - & - & - & - & - & - \\
\hline Rifampin & $\leftrightarrow$ & - & - & - & - & - & & - & - & - \\
\hline CNS agents & & & & & & & - & & & \\
\hline Buprenorphine & - & - & - & $\begin{array}{c}\uparrow 37 \\
\text { buprenorphine; } \\
\uparrow 62 \\
\text { norbuprenorphine }\end{array}$ & $\begin{array}{c}\uparrow 107 \\
\text { buprenorphine; } \\
\uparrow 84 \\
\text { norbuprenorphine }\end{array}$ & $\leftrightarrow$ & $\leftrightarrow$ & - & - & - \\
\hline
\end{tabular}

CNS, central nervous system; DCV, daclatasvir; DSV, dasabuvir; EBR, elbasvir; GLE, glecaprevir; GZR, grazoprevir; LDV, ledipasvir; LNG, levonorgestrel; NG, norgestrel; NGMN,

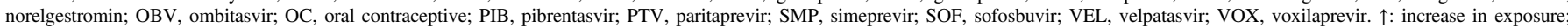
$\downarrow$ : decrease in exposure; $\leftrightarrow$ : no change; —: data not available.

${ }^{a}$ Interactions with amiodarone not identified although adverse events have been observed during coadministration with DAAs.

${ }^{b}$ SOF/VEL/VOX plus VOX.

c400/120 mg GLE/PIB daily.

DDI liability for sofosbuvir and ledipasvir (both P-gp/BCRP substrates) is limited to transporters. Coadministration of sofosbuvir with a potent $\mathrm{P}$-gp/BCRP inhibitor cyclosporine $(600 \mathrm{mg})$ increased the sofosbuvir AUC and $C_{\max }$ by $353 \%$ and $154 \%$, respectively, without an alteration in the GS-331007 AUC. Of note, at clinically relevant doses of cyclosporin, sofosbuvir exposure was minimally altered (AUC 15\% higher) with cyclosporine-based immunosuppressant regimens in posttransplant patients (Kirby et al., 2015). The effect of transporter inhibition on the pharmacokinetics of ledipasvir was assessed after coadministration of ledipasvir and the P-gp/OATP inhibitor atazanavir (ATV) plus ritonavir, resulting in a higher ledipasvir AUC $(96 \%)$ and $C_{\text {max }}(68 \%$ ) (German et al., 2015; Harvoni, 2017b).

Velpatasvir is metabolized by CYP3A and is a substrate for several drug transporters, including P-gp, BCRP, and OATP. Based on ketoconazole-mediated CYP3A/P-gp inhibition, velpatasvir AUC and $C_{\max }$ values were $70 \%$ and $29 \%$ higher, respectively, after coadministration (Mogalian et al., 2016b). Coadministration of velpatasvir with OATP1B inhibitor rifampin (single dose) resulted in a modest $(46 \%)$ increase in the velpatasvir AUC (Epclusa, 2017b).

Increases in sofosbuvir, ledipasvir, and velpatasvir pharmacokinetics were not deemed clinically relevant and the use of P450 and drug transport inhibitors was permitted in the phase III sofosbuvir, ledipasvir/sofosbuvir, and sofosbuvir/velpatasvir clinical programs. As reflected in the prescribing information, sofosbuvir, ledipasvir/ sofosbuvir, or sofosbuvir/velpatasvir may be used with P450, P-gp, BCRP, and/or OATP inhibitors (Epclusa, 2017b; Harvoni, 2017b; Sovaldi, 2017a). 
TABLE 4

Impact of HIV coadministered HIV drug or regiment on DAA exposure (AUC)

Data are given in percentages.

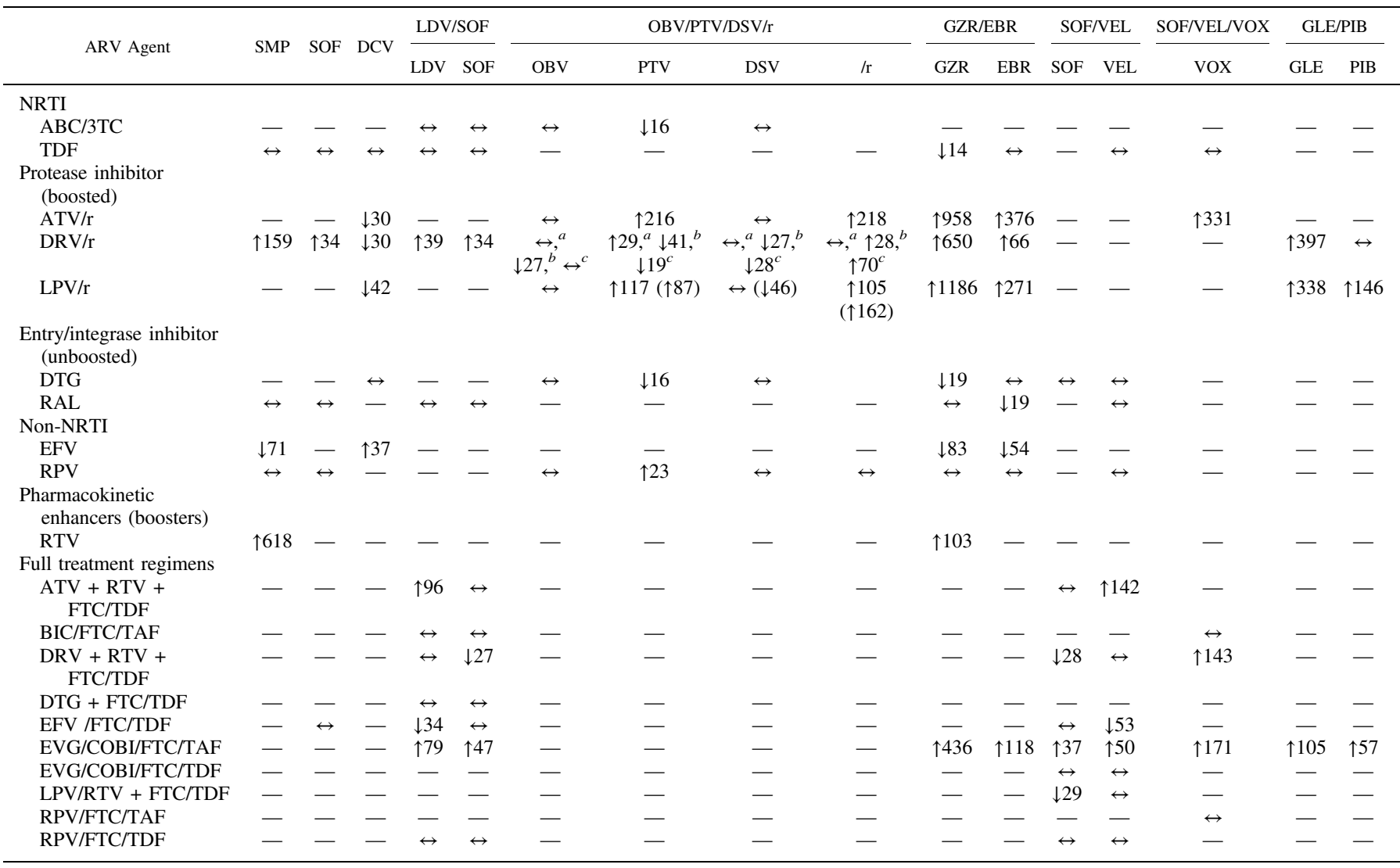

BIC, bictegravir; DCV, daclatasvir; DSV, dasabuvir; EBR, elbasvir; GLE, glecaprevir; GZR, grazoprevir; LDV, ledipasvir; OBV, ombitasvir; PIB, pibrentasvir; PTV, paritaprevir; RTV or/r, ritonavir; SMP, simeprevir; SOF, sofosbuvir; VEL, velpatasvir; VOX, voxilaprevir. $\uparrow$ : increase in exposure; $\downarrow$ : decrease in exposure; $\leftrightarrow$ : no change; —: data not available.

${ }^{a} 800 \mathrm{mg}$ DRV daily AM plus OBV/PTV/DSV/r AM versus $800 \mathrm{mg}$ daily DRV AM plus $100 \mathrm{mg}$ RTV daily AM.

${ }^{b} 600 \mathrm{mg}$ DRV twice daily AM/PM plus OBV/PTV/DSV/r AM plus $100 \mathrm{mg}$ RTV daily PM versus $600 \mathrm{mg}$ DRV twice daily AM/PM plus $100 \mathrm{mg}$ RTV twice daily AM/PM.

${ }^{c}$ OBV/PTV/DSV/r AM plus $800 \mathrm{mg}$ DRV daily PM plus $100 \mathrm{mg}$ RTV daily PM versus $800 \mathrm{mg}$ DRV daily PM plus $100 \mathrm{mg}$ RTV daily PM.

Slow turnover of voxilaprevir, primarily by CYP3A, has been observed and voxilaprevir is also a substrate for several drug transporters, including P-gp, BCRP, and OATP1B1/3. Voriconazolemediated CYP3A inhibition resulted in an $84 \%$ higher voxilaprevir AUC, with no change in $C_{\max }$ (Kirby et al., 2016a). Coadministration of voxilaprevir with combined P-gp/BCRP/OATP inhibitor cyclosporine $\left(600 \mathrm{mg}\right.$ ) resulted in $840 \%$ and $1800 \%$ higher AUC and $C_{\max }$, respectively, where the majority of the effect was attributed to OATP inhibition based on the results of coadministration of voxilaprevir with the OATP1B inhibitor rifampin (single dose; 691\% and 1010\% higher AUC and $C_{\max }$, respectively) (Kirby et al., 2016a). Coadministration of sofosbuvir/velpatasvir/voxilaprevir with cyclosporine is not recommended.

Consistent with the role of CYP3A and P-gp in daclatasvir pharmacokinetics, daclatasvir AUC and $C_{\max }$ values were $200 \%$ and $57 \%$ higher, respectively, with ketoconazole (Daklinza, 2017). A dose reduction to $30 \mathrm{mg}$ daclatasvir is recommended with strong CYP3A inhibitors. Monitoring for daclatasvir adverse events is recommended if daclatasvir is coadministered with moderate CYP3A inhibitors (Eley et al., 2014; Daklinza, 2017, 2018).

A two-way interaction was observed between ombitasvir/paritaprevir/ritonavir plus dasabuvir and ketoconazole, as evidenced by higher plasma exposures of paritaprevir (AUC 98\%; $C_{\max } 37 \%$ ), ritonavir (AUC 57\%; $C_{\max } 27 \%$ ), dasabuvir (AUC 42\%; $C_{\max } 16 \%$ ), and ketoconazole (AUC 117\%; $C_{\max } 15 \%$ ). Ketoconazole did not alter ombitasvir pharmacokinetics, in which P-gp play a role (Menon et al., 2015). Based on these collective data, the maximum daily dose of ketoconazole should be limited to $200 \mathrm{mg} /$ day during coadministration with ombitasvir/paritaprevir/ritonavir plus dasabuvir (Viekira Pak, 2017).

Dasabuvir is primarily metabolized by CYP2C8. Administration of dasabuvir with potent CYP2C8 inhibitor gemfibrozil resulted in a higher dasabuvir AUC (1025\%) and $C_{\max }(101 \%)$, respectively. Higher dasabuvir exposures were associated with QT prolongation; thus, use of strong inhibitors of CYP2C8 with ombitasvir/paritaprevir/ritonavir plus dasabuvir is contraindicated (Viekira Pak, 2017).

Elbasvir and grazoprevir are substrates of P-gp and CYP3A, and grazoprevir is also a substrate of OATP1B1/3. Coadministration of elbasvir/grazoprevir with P-gp inhibitors is expected to have minimal effect, because the role of intestinal P-gp on the absorption of elbasvir and grazoprevir appears to be minimal (Zepatier, 2018). Coadministration of elbasvir/grazoprevir with ketoconazole resulted in higher AUC and $C_{\max }$ values for elbasvir (80\% and $29 \%$, respectively) and grazoprevir (202\% and 13\%, respectively) (Zepatier, 2018). The use of strong CYP3A inhibitors with elbasvir/grazoprevir is not recommended. Coadministration of elbasvir/grazoprevir and cyclosporine (400 mg) resulted in higher AUC and $C_{\max }$ values for elbasvir (98\% and 95\%, respectively) and grazoprevir (1421\% and $1600 \%$, 
TABLE 5

Impact of DAA on coadministered HIV drug exposure (AUC)

Data are given in percentages.

\begin{tabular}{|c|c|c|c|c|c|c|c|c|c|c|}
\hline \multirow[b]{2}{*}{ ARV Agent } & \multirow[b]{2}{*}{ SMP } & \multirow[b]{2}{*}{ SOF } & \multirow[b]{2}{*}{ DCV } & \multirow[b]{2}{*}{ LDV/SOF } & \multirow[b]{2}{*}{ OBV/PTV/DSV/r } & \multicolumn{2}{|c|}{ GZR/EBR } & \multirow[b]{2}{*}{ SOF/VEL } & \multirow[b]{2}{*}{ SOF/VEL/VOX } & \multirow[b]{2}{*}{ GLE/PIB } \\
\hline & & & & & & GZR & EBR & & & \\
\hline \multicolumn{11}{|l|}{ NRTI } \\
\hline $\mathrm{ABC}$ & - & - & - & $\leftrightarrow$ & $\leftrightarrow$ & \multirow{2}{*}{\multicolumn{2}{|c|}{$\overline{-}_{\leftrightarrow^{b}}$}} & - & - & $\leftrightarrow^{b}$ \\
\hline FTC & - & $\leftrightarrow$ & - & $\leftrightarrow$ & - & & & $\leftrightarrow^{b}$ & $\leftrightarrow^{b}$ & - \\
\hline $3 \mathrm{TC}$ & - & - & - & & - & - & - & - & - & - \\
\hline TAF (10 mg, boosted $)$ & - & - & - & $\leftrightarrow^{b, d}$ & - & - & - & $\leftrightarrow^{b, d}$ & $\leftrightarrow^{b, d}$ & $\leftrightarrow^{b, d}$ \\
\hline TAF (25 mg, nonboosted) & - & - & - & - & - & - & - & - & $\uparrow 52-57^{b}$ & - \\
\hline TDF (boosted regimen) & - & - & - & $\leftrightarrow, \uparrow 50^{b, c}$ & - & \multicolumn{2}{|c|}{$\leftrightarrow^{b, d}$} & $\leftrightarrow, \uparrow 39^{b, c}$ & $\uparrow 39^{b, c}$ & - \\
\hline TDF (nonboosted regimen) & $\leftrightarrow^{a}$ & $\leftrightarrow^{b}$ & - & $\uparrow 98^{a}$ & - & & $\rightarrow{ }^{a}$ & $\uparrow 40-\uparrow 81^{b}$ & - & $\leftrightarrow^{b}$ \\
\hline \multicolumn{11}{|l|}{ Protease inhibitor } \\
\hline ATV & - & - & - & $\leftrightarrow^{b, c}$ & $\leftrightarrow^{a, c}$ & $\uparrow 43^{a, c}$ & $\leftrightarrow^{a, c}$ & $\leftrightarrow^{b, c}$ & - & - \\
\hline DRV & $\leftrightarrow^{a, c}$ & $\leftrightarrow$ & $\leftrightarrow^{a, c}$ & $\leftrightarrow^{b, c}$ & $\downarrow 24,,^{a, e} \leftrightarrow,,^{a, f} \uparrow 34^{a, g}$ & $\leftrightarrow^{a, c, h}$ & $\leftrightarrow^{a, c, h}$ & $\leftrightarrow^{b, c}$ & $\leftrightarrow^{b, c}$ & $\leftrightarrow^{b, c}$ \\
\hline LPV & - & - & $\leftrightarrow^{a, c}$ & - & $\leftrightarrow^{a, c}$ & $\leftrightarrow^{a, c}$ & $\leftrightarrow^{a, c}$ & $\leftrightarrow^{i}$ & - & - \\
\hline \multicolumn{11}{|l|}{ Entry/integrase inhibitor } \\
\hline BIC & & & - & $\leftrightarrow^{b}$ & - & - & - & - & $\leftrightarrow^{b}$ & - \\
\hline DTG & - & - & ${ }^{\uparrow} 33^{a}$ & $\leftrightarrow$ & $\uparrow 38$ & \multicolumn{2}{|c|}{$\leftrightarrow^{a}$} & $\leftrightarrow$ & - & - \\
\hline EVG & - & - & - & $\leftrightarrow^{b, d}$ & - & \multicolumn{2}{|c|}{$\leftrightarrow^{b}$} & $\leftrightarrow^{b, d}$ & $\leftrightarrow^{b, d}$ & $\leftrightarrow^{b, d}$ \\
\hline RAL & $\leftrightarrow^{a}$ & $\leftrightarrow$ & - & $\leftrightarrow$ & - & $\uparrow 43^{d}$ & $\leftrightarrow^{a}$ & $\leftrightarrow^{b}$ & - & $\uparrow 47^{a}$ \\
\hline \multicolumn{11}{|l|}{ Non-NRTI } \\
\hline EFV (600 mg daily) & $\leftrightarrow^{a}$ & $\leftrightarrow$ & - & $\leftrightarrow$ & - & \multirow{2}{*}{\multicolumn{2}{|c|}{$\leftrightarrow \leftrightarrow^{a} \leftrightarrow$}} & $\leftrightarrow^{b}$ & - & - \\
\hline RPV (25 mg daily) & $\leftrightarrow^{a}$ & $\leftrightarrow$ & - & $\leftrightarrow$ & $\uparrow 225^{a}$ & & & $\leftrightarrow^{b}$ & $\leftrightarrow^{b}$ & $\uparrow 84^{a}$ \\
\hline \multicolumn{11}{|c|}{ Pharmacokinetic enhancers (boosters) } \\
\hline COBI & - & - & - & $\uparrow 53$ & - & \multicolumn{2}{|c|}{$\uparrow 49$} & $\leftrightarrow^{b}$ & $\uparrow 50$ & - \\
\hline RTV & $\leftrightarrow$ & - & - & $\leftrightarrow$ & - & - & - & $\leftrightarrow^{b}$ & $\uparrow 45$ & $\uparrow 87$ \\
\hline
\end{tabular}

BIC, bictegravir; DCV, daclatasvir; DSV, dasabuvir; EBR, elbasvir; GLE, glecaprevir; GZR, grazoprevir; LDV, ledipasvir; OBV, ombitasvir; PIB, pibrentasvir; PTV, paritaprevir; RTV or/r, ritonavir; SMP, simeprevir; SOF, sofosbuvir; VEL, velpatasvir; VOX, voxilaprevir. $\uparrow$ : increase in exposure; $\downarrow$ : decrease in exposure; $\leftrightarrow$ : no change; - : data not available.

${ }^{a} \mathrm{HIV}$ drug tested as a single agent.

${ }^{b} \mathrm{HIV}$ drug tested as part of antiretroviral therapy regimen.

${ }^{c}$ Boosted with $100 \mathrm{mg}$ RTV daily.

${ }^{d}$ Boosted with $150 \mathrm{mg}$ COBI daily.

${ }^{e} 800 \mathrm{mg}$ DRV daily AM plus OBV/PTV/DSV/r AM versus $800 \mathrm{mg}$ DRV daily AM plus $100 \mathrm{mg}$ RTV daily AM.

${ }^{f} 600 \mathrm{mg}$ DRV twice daily AM/PM plus OBV/PTV/DSV/r AM plus $100 \mathrm{mg}$ RTV daily PM versus $600 \mathrm{mg}$ DRV twice daily AM/PM plus $100 \mathrm{mg}$ RTV twice daily AM/PM.

${ }^{g}$ OBV/PTV/DSV/r AM plus $800 \mathrm{mg}$ DRV daily PM plus $100 \mathrm{mg}$ RTV daily PM versus $800 \mathrm{mg}$ DRV daily PM plus $100 \mathrm{mg}$ RTV daily PM.

${ }^{h} 600 / 100 \mathrm{mg}$ twice daily.

${ }^{i} 800 / 200 \mathrm{mg}$ daily.

respectively), due to inhibition of P-gp, OATP1B1/3, and CYP3A (Zepatier, 2018). Coadministration of OATP1B1/3 inhibitors with grazoprevir/elbasvir is contraindicated due to significantly higher grazoprevir concentrations.

Glecaprevir and pibrentasvir are substrates of P-gp and/or BCRP, and glecaprevir is also a substrate of OATP1B1/3. Coadministration of P-gp and BCRP inhibitors may increase glecaprevir and pibrentasvir plasma exposures, whereas coadministration of OATP1B1/3 inhibitors will increase glecaprevir plasma exposures. Coadministration of cyclosporine $(400 \mathrm{mg})$, an inhibitor of P-gp/BCRP/OATP1B, resulted in a higher glecaprevir AUC (408\%) and $C_{\max }(351 \%)$ and a higher pibrentasvir AUC (93\%) with no change in $C_{\max }$ (Mavyret, 2017; Maviret, 2018). Coadministration of single-dose rifampin, an OATP1B inhibitor, resulted in a higher glecaprevir AUC (755\%) and $C_{\max }(552 \%)$ with no change in pibrentasvir exposure (Mavyret, 2017; Maviret, 2018). Regional prescribing information for glecaprevir/pibrentasvir should be consulted for specific recommendations on the coadministration of various P-gp, BCRP, and/or OATP1B inhibitors (Mavyret, 2017; Maviret, 2018).

DAAs as Perpetrators Metabolizing Enzyme-Mediated Interactions. The potential of simeprevir to perpetrate P450-mediated DDIs was evaluated using a metabolic cocktail approach. Simeprevir demonstrated mild inhibition of CYP1A2 (caffeine AUC 26\% higher) and intestinal CYP3A (oral midazolam AUC $45 \%$ higher; $C_{\max } 31 \%$ higher) with no alteration in exposure after intravenous midazolam (Sekar et al., 2010). No effect on CYP2C9, CYP2C19, or CYP2D6 was observed (Olysio, 2017).
Sofosbuvir, ledipasvir, velpatasvir, and elbasvir are not expected to perpetrate any metabolic enzyme-mediated DDIs. Although daclatasvir may induce CYP3A, several clinical studies with coadministered CYP3A substrates have demonstrated that daclatasvir is unlikely to elicit clinically relevant interactions (Garimella et al., 2016).

The exposure of omeprazole, a substrate of CYP2C19, was 38\% lower when coadministered with ombitasvir/paritaprevir/ritonavir plus dasabuvir, revealing an inductive effect of the DAA regimen on CYP2C19 and possibly warranting adjustment of the omeprazole dose in patients whose symptoms are poorly controlled (Menon et al., 2015). Use of sensitive CYP3A substrates with ombitasvir/paritaprevir/ritonavir plus dasabuvir is contraindicated due to the presence of potent CYP3A inhibitor ritonavir within the regimen (Viekira Pak, 2017). Ombitasvir, paritaprevir, and dasabuvir are also inhibitors of UGT1A1 and may result in higher plasma concentrations of drugs that are UGT1A1 substrates (e.g., levothyroxine; see label) (Viekira Pak, 2017).

\section{Transporter-Mediated Interactions}

P-gp (Digoxin or Dabigatran Etexilate as Probe Substrates). Digoxin and dabigatran etexilate are P-gp substrates that help to assess P-gp-mediated DDIs. Dabigatran etexilate is primarily sensitive to P-gp intestinally, and digoxin is sensitive to P-gp both intestinally and systemically. Overall digoxin exposure (AUC) was modestly higher after coadministration with simeprevir, daclatasvir, ledipasvir, or velpatasvir (AUC 27\%-34\% higher) and was 11\% higher with elbasvir (Eley et al., 2011; German et al., 2014b; Mogalian et al., 2016b; Ouwerkerk-Mahadevan et al., 2016; Daklinza, 2017; Zepatier, 2018). 
Administration of digoxin with ombitasvir/paritaprevir/ritonavir plus dasabuvir resulted in no alteration to digoxin exposure despite in vitro P-gp inhibition by ritonavir, paritaprevir, and dasabuvir (Menon et al., 2015). Due to the narrow therapeutic index of digoxin, caution is warranted and therapeutic monitoring may be required during coadministration with simeprevir, daclatasvir, ledipasvir/sofosbuvir, or sofosbuvir/velpatasvir. Per the prescribing information, no dose adjustment for digoxin is required for coadministration with ombitasvir/ paritaprevir/ritonavir plus dasabuvir or elbasvir/grazoprevir (Viekira Pak, 2017; Zepatier, 2018).

Dabigatran exposure (AUC) was $161 \%$ higher after coadministration with sofosbuvir/velpatasvir/voxilaprevir and was $138 \%$ higher upon coadministration with glecaprevir/pibrentasvir (Mavyret, 2017; Vosevi, 2017a,b; Maviret, 2018). Regional prescribing information for sofosbuvir/velpatasvir/voxilaprevir and glecaprevir/pibrentasvir should be consulted for specific recommendations on coadministration with dabigatran etexilate.

OATP1B and BCRP (Pravastatin and Rosuvastatin as Probe Substrates). 3-Hydroxy-3-methylglutaryl-CoA reductase inhibitors (statins) are commonly used as probes to assess OATP- and/or BCRPmediated DDIs clinically. The hepatic uptake of most statins, including pravastatin and rosuvastatin, is mediated by OATP1B1 (Neuvonen et al., 2006). Rosuvastatin is also transported by BCRP (and sodiumtaurocholate cotransporting polypeptide) (Neuvonen et al., 2006).

Rosuvastatin exposure was higher after administration with simeprevir (AUC 181\%; $C_{\max } 217 \%$ ) and with daclatasvir (AUC 58\%; $C_{\max }$ $104 \%$ ) due to inhibition of OATP and BCRP (Daklinza, 2014, 2017; Olysio, 2017). Caution and appropriate monitoring for statin-related adverse events is recommended on coadministration of rosuvastatin with simeprevir or daclatasvir; for simeprevir, the dose of rosuvastatin should be initiated at $5 \mathrm{mg}$ once daily and should not exceed $10 \mathrm{mg}$ once daily (Olysio, 2017).

OATP- and BCRP-associated DDI liabilities were not directly assessed for ledipasvir. In the absence of definitive clinical data, the use of rosuvastatin with ledipasvir/sofosbuvir is not recommended (Harvoni, 2017a). Pravastatin can be used with ledipasvir/sofosbuvir without dose modification (Harvoni, 2017b). Administration of velpatasvir resulted in higher AUC and $C_{\max }$ values for pravastatin (35\% and $28 \%$, respectively) and rosuvastatin (169\% and $161 \%$, respectively). Pravastatin can be used without dose modification and the dose of rosuvastatin should not exceed $10 \mathrm{mg}$ once daily when coadministered with sofosbuvir/velpatasvir (Mogalian et al., 2016b).

With ombitasvir/paritaprevir/ritonavir plus dasabuvir, coadministered pravastatin resulted in higher pravastatin AUC $(82 \%)$ and $C_{\max }(37 \%)$, and coadministered rosuvastatin resulted in higher rosuvastatin AUC (159\%) and $C_{\max }(613 \%)$ (Viekira Pak, 2017). The daily doses of pravastatin and rosuvastatin should not exceed 40 and $10 \mathrm{mg}$, respectively, when administered with ombitasvir/paritaprevir/ritonavir plus dasabuvir (Viekira Pak, 2017).

Coadministration of elbasvir/grazoprevir with pravastatin resulted in no clinically relevant change to pravastatin AUC (33\% higher) or $C_{\max }$ (28\% higher), whereas coadministration with rosuvastatin resulted in higher rosuvastatin AUC (126\%) and $C_{\max }$ (449\%) (Zepatier, 2018). The rosuvastatin dose should not exceed $10 \mathrm{mg}$ once daily when coadministered with elbasvir/grazoprevir (Zepatier, 2018).

Coadministration of sofosbuvir/velpatasvir/voxilaprevir with pravastatin resulted in higher pravastatin AUC (116\%) and $C_{\max }(89 \%)$, whereas coadministration with rosuvastatin resulted in higher rosuvastatin AUC (639\%) and $C_{\max }(1788 \%)$ (Vosevi, 2017a,b). The daily dose of pravastatin should not exceed $40 \mathrm{mg}$ when administered with sofosbuvir/velpatasvir/voxilaprevir, whereas rosuvastatin should not be coadministered.
Numerous studies were conducted on the coadministration of glecaprevir/pibrentasvir with statins. Coadministration of glecaprevir/ pibrentasvir resulted in higher exposures for atorvastatin (AUC 728\%; $C_{\max } 2100 \%$ ), simvastatin (AUC 132\%; $C_{\max } 99 \%$; simvastatin acid AUC 348\%; $C_{\max }$ 970\%), lovastatin (AUC 70\%; lovastatin acid AUC $310 \%$; $C_{\max } 473 \%$ ), pravastatin (AUC 130\%; $C_{\max } 123 \%$ ), and rosuvastatin (AUC $115 \%$; $C_{\max } 462 \%$ ). Coadministration of atorvastatin, lovastatin, and simvastatin with glecaprevir/pibrentasvir is not recommended. If glecaprevir/pibrentasvir is coadministered with pravastatin, the pravastatin dose should be reduced by $50 \%$. The rosuvastatin dose should not exceed $10 \mathrm{mg}$ when coadministered with glecaprevir/pibrentasvir.

In summary, caution and appropriate monitoring for statin-, digoxin-, or dabigatran-related adverse events has been recommended for DAAs identified or expected to cause transporter (or CYP3A)-mediated interactions. In specific cases, coadministration is not recommended or dosing recommendations are specified within the prescribing information. These data also inform potential for interactions with other sensitive substrates of these drug transporters.

\section{Other Commonly Used Medications in HCV}

\section{Methadone and Buprenorphine}

Opioid agonists, methadone and buprenorphine, are frequently prescribed in the HCV-infected population to treat opioid dependence. No clinically meaningful interactions were observed when simeprevir, sofosbuvir, daclatasvir, ombitasvir/paritaprevir/ritonavir plus dasabuvir, elbasvir/grazoprevir, or glecaprevir/pibrentasvir were administered to patients receiving opioid therapy with methadone (CYP3A and CYP2B6 substrate), whereas daclatasvir or elbasvir/grazoprevir did not impact the pharmacokinetics of buprenorphine (CYP3A and CYP2C8 substrate) (Daklinza, 2017; Olysio, 2017; Sovaldi, 2017a; Viekira Pak, 2017; Zepatier, 2018). Ledipasvir/sofosbuvir, sofosbuvir/ velpatasvir, and sofosbuvir/velpatasvir/voxilaprevir do not inhibit or induce enzymes involved in the disposition of methadone or buprenorphine; thus, DDI studies with these agents were not conducted. Coadministration of ombitasvir/paritaprevir/ritonavir plus dasabuvir resulted in higher AUC and $C_{\max }$ values for buprenorphine (107\% and $118 \%$, respectively) and norbuprenorphine (major active metabolite; $84 \%$ and $107 \%$, respectively) (Menon et al., 2015). Although no buprenorphine dose adjustment is necessary, close monitoring of patients for sedation and cognitive effects during coadministration with ombitasvir/paritaprevir/ritonavir plus dasabuvir is warranted (Viekira Pak, 2017).

\section{Immunosuppressants}

Recurrence of HCV infection is universal in viremic patients after liver transplantation, and treatment of $\mathrm{HCV}$ infection in post-transplant patients is associated with substantial improvements in both patient and graft survival (Suraweera et al., 2016). Calcineurin inhibitors tacrolimus and cyclosporine are commonly used to prevent graft rejection.

Cyclosporine. Cyclosporine is a substrate of CYP3A, P-gp, and OATP1B and a strong inhibitor of CYP3A, P-gp, BCRP, and OATP1B (Prograf, 2003; Krishna et al., 2007; Kalliokoski and Niemi, 2009; Neoral, 2009). A wide range of cyclosporine doses were used to assess DDI susceptibility with DAAs, ranging from low doses $(30 \mathrm{mg}$ ) or higher doses (up to $600 \mathrm{mg}$ ) that are more typical of doses after organ transplantation.

Higher cyclosporine AUC (19\%) and $C_{\max }(16 \%)$ was observed after coadministration of cyclosporine $(400 \mathrm{mg}$ ) with simeprevir (OuwerkerkMahadevan et al., 2012c). Results from a phase II study of simeprevir in combination with daclatasvir and ribavirin in $\mathrm{HCV}$-infected post-liver 
transplant patients showed higher simeprevir AUC (468\%) and $C_{\max }$ $(353 \%)$ with cyclosporine (Ouwerkerk-Mahadevan et al., 2016; Olysio, 2017); coadministration of cyclosporine and simeprevir is not recommended due to the significantly higher simeprevir exposure (Olysio, 2017).

Cyclosporine (600 mg)-mediated inhibition of P-gp/BCRP resulted in higher sofosbuvir AUC $(353 \%)$ and $C_{\max }(154 \%)$ values without altering GS-331007 exposure; no sofosbuvir dose adjustment is required during use with cyclosporine (Mathias et al., 2012). Similarly, no dose adjustment is required for ledipasvir or velpatasvir (AUC 103\% higher; $C_{\max } 56 \%$ higher) when coadministered with cyclosporine (Mogalian et al., 2016b). Cyclosporine may be administered with sofosbuvir, ledipasvir/sofosbuvir, or sofosbuvir/velpatasvir. As discussed above, the strong inhibition of OATP1B by cyclosporine $(600 \mathrm{mg})$ was primarily responsible for the higher voxilaprevir AUC (839\%) and $C_{\max }$ (1802\%) observed after coadministration with sofosbuvir/velpatasvir/ voxilaprevir. Coadministration of cyclosporine with sofosbuvir/ velpatasvir/voxilaprevir is not recommended (Vosevi, 2017a).

A higher daclatasvir AUC (40\%) was observed with cyclosporine (400 mg), with no change in cyclosporine pharmacokinetics, supporting coadministration of cyclosporine with daclatasvir (Bifano et al., 2013).

The cyclosporine AUC was $482 \%$ higher with no alteration in $C_{\max }$ when administered with ombitasvir/paritaprevir/ritonavir plus dasabuvir (Badri et al., 2015; Viekira Pak, 2017). The effect of ombitasvir/ paritaprevir/ritonavir plus dasabuvir on cyclosporine pharmacokinetics is consistent with OATP and CYP3A inhibition by paritaprevir and ritonavir, respectively. Based on these results, specific dosing recommendations were warranted and are discussed in more detail in the USPI and SmPC (Viekira Pak, 2017; Viekirax, 2018). The effects of low-dose (30 mg) cyclosporine on ombitasvir/paritaprevir/ritonavir plus dasabuvir pharmacokinetics resulted in higher paritaprevir AUC (72\%), lower dasabuvir AUC (30\%), and no change in the exposure of ombitasvir or ritonavir.

As discussed above, coadministration of elbasvir/grazoprevir with cyclosporine is contraindicated due to significantly higher simeprevir AUC $(1421 \%)$ and $C_{\max }(1600 \%)$ after administration with cyclosporine $400 \mathrm{mg}$ (Zepatier, 2018).

Coadministration of glecaprevir/pibrentasvir with two different doses of cyclosporine (100 or $400 \mathrm{mg}$ ) was studied. At the higher cyclosporine dose, coadministration resulted in higher glecaprevir AUC (408\%) and $C_{\max }(351 \%)$ and higher pibrentasvir AUC $(93 \%)$ with no change in $C_{\max }$ (Mavyret, 2017; Maviret, 2018). At the lower cyclosporine dose, the glecaprevir AUC was $37 \%$ higher $\left(C_{\max } 30 \%\right.$ higher), with no change in pibrentasvir exposure. Coadministration of glecaprevir/ pibrentasvir with stable cyclosporine doses $>100 \mathrm{mg} /$ day is not recommended (Mavyret, 2017).

Tacrolimus. Tacrolimus is a substrate of CYP3A and P-gp, although it is more sensitive to P450-mediated drug interactions (Prograf, 2003; Krishna et al., 2007; Kalliokoski and Niemi, 2009; Neoral, 2009). Tacrolimus is also an inhibitor of CYP3A and P-gp.

No alteration in tacrolimus exposure was observed after coadministration with simeprevir (Ouwerkerk-Mahadevan et al., 2012c). Results from a phase II study of simeprevir in combination with and ribavirin in HCV-infected post-liver transplant patients showed higher simeprevir AUC $(90 \%)$ and $C_{\max }(85 \%)$ with tacrolimus; no dose adjustment is required when simeprevir is administered with tacrolimus (OuwerkerkMahadevan et al., 2016; Olysio, 2017).

No clinically relevant interactions were observed between tacrolimus and sofosbuvir or daclatasvir (Bifano et al., 2013; Daklinza, 2017). Based on the DDI profiles for ledipasvir, velpatasvir, voxilaprevir, and tacrolimus, clinically significant interactions are not expected and thus the DDI studies were not conducted. Tacrolimus may be administered with sofosbuvir, ledipasvir/sofosbuvir, sofosbuvir/velpatasvir, sofosbuvir/ velpatasvir/voxilaprevir, or daclatasvir (Epclusa, 2017b; Harvoni, 2017b; Sovaldi, 2017a; Vosevi, 2017a).

Tacrolimus AUC and $C_{\max }$ values were $5613 \%$ and $299 \%$ higher, respectively, when administered with ombitasvir/paritaprevir/ritonavir plus dasabuvir (Badri et al., 2015; Viekira Pak, 2017); the effect of ombitasvir/paritaprevir/ritonavir plus dasabuvir on tacrolimus pharmacokinetics is consistent with strong CYP3A inhibition by ritonavir. Based on these data, the use of tacrolimus (as well as sirolimus and everolimus) is contraindicated with ombitasvir/paritaprevir/ritonavir plus dasabuvir (Viekira Pak, 2017; Viekirax, 2018).

The tacrolimus AUC was $43 \%$ higher when coadministered with grazoprevir, thus warranting the monitoring of tacrolimus concentrations, adverse events, and changes in renal function upon initiation of coadministration with elbasvir/grazoprevir (Zepatier, 2018).

Coadministration of glecaprevir/pibrentasvir with tacrolimus resulted in a higher tacrolimus AUC (45\%) and $C_{\max }(50 \%)$. In association with the expected increase in tacrolimus exposure, use with caution and therapeutic monitoring (including dose adjustment) of tacrolimus is recommended upon coadministration with glecaprevir/pibrentasvir (Mavyret, 2017).

\section{Oral Contraceptives}

Commonly used oral hormonal contraceptives contain a combination of estrogens (e.g., ethinyl estradiol; EE) and progestins (e.g., norethindrone, norgestimate, or levonorgestrel) that undergo extensive metabolism. EE is a substrate for P-gp/BCRP, CYP3A, UGT, and sulfotransferase (Zhang et al., 2007). Norgestimate undergoes firstpass metabolism to the primary active metabolite norelgestromin and is then metabolized to norgestrel, which has limited biologic activity, and other metabolites (Hammond et al., 2003; Olysio, 2017). HCV-infected women of childbearing potential may need to receive ribavirin (pregnancy category $\mathrm{X}$ ) with their DAA regimen, thus warranting assessment of DDIs between DAAs and oral contraceptives.

Results from DDI studies demonstrated no need for dose adjustment of evaluated oral contraceptives with simeprevir, sofosbuvir, ledipasvir/ sofosbuvir, sofosbuvir/velpatasvir, sofosbuvir/velpatasvir/voxilaprevir, daclatasvir, elbasvir/grazoprevir, or glecaprevir/pibrentasvir. Briefly, administration of simeprevir with $\mathrm{EE} /$ norethindrone increased $\mathrm{EE} C_{\max }$ (18\%), with no alteration to other parameters (Ouwerkerk-Mahadevan et al., 2012d). Coadministration of sofosbuvir, ledipasvir, or velpatasvir with EE/norgestimate resulted in a higher norgestrel AUC (19\%) with sofosbuvir or a higher $\mathrm{EE} C_{\max }$ with ledipasvir (40\%) or velpatasvir (39\%), respectively (German et al., 2014a). EE $C_{\max }$ increases with ledipasvir or velpatasvir were attributed to P-gp/BCRP inhibition; a mechanism for higher norgestrel AUC with sofosbuvir is unknown (German et al., 2014a; Mogalian et al., 2014). There was no alteration in EE or norgestimate pharmacokinetics with sofosbuvir/velpatasvir/ voxilaprevir (Vosevi, 2017a). Daclatasvir or elbasvir/grazoprevir did not alter pharmacokinetics of EE/norgestimate or EE/levonorgestrel, respectively (Bifano et al., 2014; Daklinza, 2017; Marshall et al., 2017; Zepatier, 2018). Coadministration of glecaprevir/pibrentasvir with EE/nogestimate or EE/levonorgestrel resulted in higher EE (28\%$40 \%$ ), norgestrel (63\%-68\%), and/or norelgestromin (44\%). No dose adjustment with levonorgestrel, norethnidrone, or norgestimate is needed when coadministered with glecaprevir/pibrentasvir; coadministration of glecaprevir/pibrentasvir is contraindicated with EEcontaining oral contraceptives due to risk of alanine aminotransferase (ALT) elevations.

Administration of ombitasvir/paritaprevir/ritonavir plus dasabuvir with EE/norgestimate did not significantly impact EE but resulted in higher norelgestromin AUC (160\%) and norgestrel AUC (154\%) and 
lower paritaprevir AUC (34\%), ritonavir AUC (29\%), and dasabuvir AUC (52\%) (Menon et al., 2015). The use of EE-containing oral contraceptives with ombitasvir/paritaprevir/ritonavir plus dasabuvir is contraindicated due to potential for ALT elevations during coadministration; the mechanism for this pharmacodynamic interaction is unknown (Menon et al., 2015). Coadministration of ombitasvir/ paritaprevir/ritonavir plus dasabuvir with norethindrone (progestin-only contraceptive) resulted in altered exposure for only paritaprevir (AUC 23\% higher), supporting coadministration (Viekira Pak, 2017; Viekirax, 2018).

\section{HCV/HIV Coinfection}

Approximately $25 \%$ of HIV-infected individuals in the United States are coinfected with HCV (Soriano et al., 2010; https://www.cdc.gov/ hepatitis/populations/hiv.htm and https://www.cdc.gov/hepatitis/ hepawarenessabcs.htm). Liver fibrosis progresses approximately three times more rapidly in $\mathrm{HIV} / \mathrm{HCV}$-coinfected patients than in patients with HCV monoinfection (Graham et al., 2001). Although current guidelines for ARV therapy in patients with HIV are the same regardless of HCV coinfection status, the selection of HCV DAAs must be carefully considered due to the potential for significant DDIs between treatment regimens. An important consideration in evaluating results of DDI studies between HCV DAAs and HIV ARVs is whether those studies were conducted with full treatment regimens or with only single components, as studies accounting for both intra- and inter-regimen DDIs best advise the possible magnitude, and thus clinical interpretation, of DDI results.

\section{Nucleoside/Nucleotide Reverse Transcriptase Inhibitors}

Nucleoside or nucleotide reverse transcriptase inhibitors (NRTIs), such as tenofovir disoproxil fumarate (TDF), tenofovir alafenamide (TAF), emtricitabine (FTC), abacavir (ABC), and lamivudine (3TC) are considered the backbones of HIV treatment regimens. Tenofovir disoproxil and TAF are both P-gp/BCRP substrates and prodrugs of the nucleotide analog tenofovir (TFV) (Tong et al., 2007). FTC, ABC, and 3TC are known to have a limited DDI profile. TFV, FTC, and 3TC are renally eliminated (Epivir, 2013; Truvada, 2017).

Results of DDI studies between TDF (single agent) and simeprevir, daclatasvir, or elbasvir/grazoprevir did not demonstrate clinically relevant interactions (i.e., $<30 \%$ increase in TFV exposure from TDF), supporting use of these agents together (Bifano et al., 2012; Olysio, 2017; Zepatier, 2018).

A regimen-based approach was used to assess DDIs between sofosbuvir, ledipasvir/sofosbuvir, sofosbuvir/velpatasvir, or sofosbuvir/ velpatasvir/voxilaprevir with boosted and unboosted FTC-, TDF-, and TAF-containing regimens. Administration with sofosbuvir, ledipasvir/ sofosbuvir, sofosbuvir/velpatasvir, or sofosbuvir/velpatasvir/voxilaprevir did not result in clinically relevant interactions with FTC or TAF as part of any regimen (Kirby et al., 2012; Epclusa, 2017b; Harvoni, 2017b; Sovaldi, 2017a; Vosevi, 2017a).

Coadministration of ledipasvir/sofosbuvir with TDF-containing regimens resulted in higher TFV exposures from EFV/FTC/TDF (AUC $98 \%$ higher; $C_{\max } 79 \%$ higher) and FTC/rilpivirine (RPV)/TDF (AUC $40 \%$ higher); similar increases in TFV exposure (approximately 40\%) were observed with other regimens, including ritonavir-boosted ATV or darunavir (DRV) plus FTC/TDF (Harvoni, 2017b). Coadministration of sofosbuvir/velpatasvir with boosted and unboosted TDF-containing regimens resulted in similar effects on TFV (approximately $40 \%$ higher AUC and $C_{\max }$ for all regimens except EFV/FTC/TDF; TFV AUC $81 \%$ higher) (Mogalian et al., 2015a, 2016a,c, 2018). The relatively larger changes in TFV seen with EFV/FTC/TDF compared with other regimens are due to comparatively lower TFV exposure when administered as EFV/FTC/TDF, which is recommended to be given on an empty stomach. The addition of voxilaprevir (i.e., sofosbuvir/ velpatasvir/voxilaprevir) did not further impact TFV exposures compared with those observed with sofosbuvir/velpatasvir (39\% higher AUC with DRV/r plus FTC/TDF; Vosevi, 2017a,b). The mechanism for the higher TFV exposure from TDF-containing regimens is attributed to inhibition of P-gp/BCRP by ledipasvir or velpatasvir and/or voxilaprevir. Monitoring for TFV-associated adverse events in patients receiving ledipasvir/sofosbuvir or sofosbuvir/velpatasvir or sofosbuvir/velpatasvir/ voxilaprevir with an ARV regimen containing TDF is advised (Epclusa, 2017b; Harvoni, 2017b; Vosevi, 2017a).

Administration of ombitasvir/paritaprevir/ritonavir plus dasabuvir with FTC/TDF resulted in lower paritaprevir AUC (16\%) and $C_{\max }$ $(32 \%)$ with no other relevant interactions (Khatri et al., 2014a); no dose adjustment of FTC/TDF is needed. No clinically significant interactions were observed with ombitasvir/paritaprevir/ritonavir plus dasabuvir administered with ABC/3TC (Khatri et al., 2016).

The regimen-based approach was used to assess DDIs between glecaprevir/pibrentasvir and FTC-, TDF-, and TAF-containing regimens. The TFV AUC was unchanged after coadministration of glecaprevir/pibrentasvir with elvitegravir (EVG)/cobicistat (COBI)/ FTC/TAF and was $29 \%$ higher after coadministration of glecaprevir/ pibrentasvir with EFV/FTC/TDF (Mavyret, 2017; Maviret, 2018).

\section{Non-NRTIs}

Non-NRTIs EFV and RPV are approved for use in combination with two NRTIs for the treatment of HIV (https://aidsinfo.nih.gov/guidelines/ html/1/adult-and-adolescent-arv/11/what-to-start).

Efavirenz. EFV is a CYP2B6 and CYP3A substrate and has also been shown to induce hepatic CYP2B6 and CYP3A, and as such may decrease the exposure of drugs metabolized by these enzymes (Olysio, 2017; Sustiva, 2017). Consistent with EFV-mediated CYP3A induction, lower plasma exposure of simeprevir (AUC 71\% lower) was observed after coadministration of EFV single agent (Ouwerkerk-Mahadevan et al., 2012a). A decrease in the velpatasvir AUC (53\%) was also observed after coadministration of sofosbuvir/velpatasvir with EFV/FTC/TDF, although no impact of sofosbuvir AUC was observed (Mogalian et al., 2015a). Due to a potential loss of therapeutic effect, coadministration of simeprevir or sofosbuvir/velpatasvir with EFVbased regimens is not recommended (Olysio, 2017).

Coadministration of ledipasvir/sofosbuvir with EFV/FTC/TDF resulted in 34\% lower ledipasvir AUC. The decrease was not deemed clinically relevant; the mechanism was postulated to be related to induction of P-gp and/or oxidative pathways that mediate ledipasvir disposition. Again, no change in sofosbuvir pharmacokinetics was observed with EFV/FTC/TDF (German et al., 2014c).

EFV was predicted to lower daclatasvir exposure by $50 \%$, but a clinical study of $120 \mathrm{mg}$ daclatasvir (twice the clinical dose) showed a more modest decrease (approximately 30\%) on daclatasvir exposure, thereby supporting the use of daclatasvir $90 \mathrm{mg}$ with EFV (Daklinza, 2017).

Administration of elbasvir and grazoprevir with EFV resulted in significantly lower AUC for both elbasvir (54\%) and grazoprevir (83\%); coadministration of elbasvir/grazoprevir with EFV-containing HIV ARV regimens is contraindicated (Zepatier, 2018). The use of ombitasvir/paritaprevir/ritonavir plus dasabuvir with EFV-based regimens is also contraindicated, as a DDI study with EFV/FTC/TDF resulted in premature study discontinuation due to significant gastrointestinal and neurologic adverse events and liver enzyme elevations (HCV Guidelines, 2015). No pharmacokinetic results are available from this study (Viekira Pak, 2017). 
Coadministration of glecaprevir/pibrentasvir with EFV/FTC/TDF resulted in significantly lower exposures of glecaprevir and pibrentasvir (exposure values not reported); coadministration of glecaprevir/ pibrentasvir with EFV-containing regimens is not recommended (Mavyret, 2017).

Rilpivirine. RPV is a CYP3A substrate and does not perpetrate transport- or enzyme-mediated interactions at the clinically relevant dose. RPV is available as a single agent or within fixed-dose combinations FTC/RPV/TDF or FTC/RPV/TAF.

For DAAs such as simeprevir, ledipasvir/sofosbuvir, sofosbuvir/ velpatasvir, sofosbuvir/velpatasvir/voxilaprevir, daclatasvir, and elbasvir/ grazoprevir that do not inhibit CYP3A, no clinically relevant interactions were observed in DDI studies, thereby supporting coadministration of these agents with RPV-containing regimens.

DDIs between ombitasvir/paritaprevir/ritonavir plus dasabuvir and RPV were also assessed. Coadministration of RPV with ombitasvir/ paritaprevir/ritonavir plus dasabuvir did not alter the pharmacokinetics of ritonavir, ombitasvir, or dasabuvir, whereas higher paritaprevir AUC (23\%) and $C_{\max }(30 \%)$ were observed (Khatri et al., 2014a). Significantly higher RPV AUC (225\%) and $C_{\max }(155 \%)$ were observed after administration of RPV with ombitasvir/paritaprevir/ ritonavir plus dasabuvir, attributable to CYP3A inhibition by ritonavir. Since higher concentrations of RPV may cause QTc interval prolongation (Olysio, 2017), coadministration of ombitasvir/ paritaprevir/ritonavir plus dasabuvir with RPV is not recommended (Viekira Pak, 2017).

A higher RPV AUC (84\%) was observed after administration with glecaprevir/pibrentasvir, although it was not considered clinically relevant and supports coadministration with RPV-containing regimens (Mavyret, 2017).

\section{Protease Inhibitors}

Protease inhibitor-based regimens for HIV containing ATV, DRV, or lopinavir (LPV) have demonstrated virologic potency and a high genetic barrier to resistance (https://aidsinfo.nih.gov/guidelines/html/1/adultand-adolescent-arv/11/what-to-start). These protease inhibitors are CYP3A substrates and are administered with a pharmacokinetic booster (ritonavir $[/ \mathrm{r}]$ or $\mathrm{COBI}[/ \mathrm{c}]$ ) to increase systemic exposure (i.e., ATV/r or ATV/c, DRV/r or DRV/c, LPV/r). ATV/r inhibits CYP3A, UGT1A1, P-gp/BCRP, and OATP1B1/1B3 (Reyataz, 2017). DRV/r inhibits CYP3A, CYP2D6, and P-gp (Olysio, 2017). LPV/r inhibits CYP3A, P-gp, and OATP1B1/3 (http://www.fda.gov/downloads/Drugs/ GuidanceComplianceRegulatoryInformation/Guidances/UCM292362. pdf). Since protease inhibitor-based regimens are administered to $\mathrm{HCV} / \mathrm{HIV}$ coinfected patients and have a higher potential for interactions than other ARV classes, assessment of DDIs is needed to inform dosing recommendations.

After administration of DRV/r with simeprevir, a higher DRV $C_{\min }$ (31\%) and ritonavir AUC (32\%) and $C_{\max }(23 \%)$ were observed compared with DRV/r alone (Ouwerkerk-Mahadevan et al., 2012b). Simeprevir exposure was significantly higher (AUC 159\%) after coadministration with DRV/r compared with $150 \mathrm{mg}$ simeprevir alone. Use of simeprevir with any HIV protease inhibitor is not recommended (Olysio, 2017).

Administration of sofosbuvir with DRV/r resulted in higher sofosbuvir AUC (34\%) and $C_{\max }(45 \%)$, with no change in DRV, ritonavir, or GS-331007 exposure. The higher sofosbuvir exposure was likely mediated by ritonavir-mediated P-gp/BCRP inhibition (Kirby et al., 2012). DDI assessment with ledipasvir/sofosbuvir and boosted protease inhibitors was conducted using complete HIV regimens of ATV/r or DRV/r plus FTC/TDF (German et al., 2015). The ledipasvir AUC was higher $(96 \%)$ when coadministered with ATV/r plus FTC/TDF, likely due to ATV/r-mediated P-gp/BCRP inhibition. The sofosbuvir AUC was unchanged when ledipasvir/sofosbuvir was administered with ATV/r plus FTC/TDF, likely due to existing P-gp/BCRP inhibition by ledipasvir as part of ledipasvir/sofosbuvir. ATV and ritonavir $C_{\min }$ values were $63 \%$ and $45 \%$ higher, respectively, when administered with ledipasvir/sofosbuvir. Administration of ledipasvir/sofosbuvir with a DRV/r-based regimen resulted in modestly lower sofosbuvir AUC (27\%) and $C_{\max }(37 \%)$ with no alteration in GS-331007, ledipasvir, DRV, or ritonavir AUC.

Administration of sofosbuvir/velpatasvir with FTC/TDF plus ATV/r, $\mathrm{DRV} / \mathrm{r}$, or LPV/r resulted in a higher velpatasvir AUC (142\%) after administration with ATV/r, attributable to P-gp/BCRP and CYP3A inhibition. No change in the velpatasvir AUC was observed when administered with DRV/r or LPV/r. The overall exposure of ATV, DRV, LPV, and ritonavir was unaffected after administration with sofosbuvir/ velpatasvir, supporting coadministration of these regimens (Epclusa, 2017b).

Coadministration of sofosbuvir/velpatasvir/voxilaprevir with DRV plus ritonavir plus FTC/TDF resulted in a higher voxilaprevir AUC (143\%) and $C_{\max }(72 \%)$, supporting coadministration. Coadministration of sofosbuvir/velpatasvir/voxilaprevir with a single dose of ATV/r resulted in a relatively greater effect (voxilaprevir AUC and $C_{\max }$ values were $331 \%$ and $342 \%$ higher, respectively) due to comparatively more potent OATP inhibition as well as P-gp/BCRP and CYP3A inhibition; as such, coadministration with ATV/r is not recommended.

A reduced dose of daclatasvir $(20 \mathrm{mg})$ was used to study the interaction between daclatasvir and ATV/r, which resulted in lower daclatasvir AUC $(30 \%)$ and $C_{\max }(55 \%)$ with $\mathrm{ATV} / \mathrm{r}$ relative to the standard daclatasvir dose $(60 \mathrm{mg}$ ) alone; there was no change in ATV/r pharmacokinetics. Based on these data, $30 \mathrm{mg}$ daclatasvir is recommended when used with ATV/r (strong CYP3A inhibitors); coadministration of $60 \mathrm{mg}$ daclatasvir with DRV/r or unboosted ATV (moderate CYP3A inhibitors) warrants monitoring for daclatasvir adverse events (Bifano et al., 2012; Daklinza, 2017).

When ombitasvir/paritaprevir/ritonavir plus dasabuvir was administered simultaneously with ATV, paritaprevir AUC was 94\% higher, whereas ombitasvir and dasabuvir pharmacokinetics were unchanged. Paritaprevir is administered with ritonavir to boost its exposure; but as demonstrated in this DDI, paritaprevir is susceptible to further increase in the presence of a second CYP3A and/or OATP inhibitor like ATV. Per the prescribing information, coadministration of ATV with ombitasvir/ paritaprevir/ritonavir plus dasabuvir should only be given in the morning (USPI; Viekira Pak, 2017) or if administered at the same time (SmPC; Viekirax, 2018). Exposures of all DAAs were lower after administration of ombitasvir/paritaprevir/ritonavir plus dasabuvir with DRV/r twice daily, and DRV $C \tau$ was also $43 \%$ to $46 \%$ lower. Coadministration of DRV/r with ombitasvir/paritaprevir/ritonavir plus dasabuvir is not recommended per the USPI; in the European Union, DRV may be administered at the same time as ombitasvir/paritaprevir/ritonavir plus dasabuvir (Viekirax, 2018). Administration of LPV/r either together with, or staggered 12 hours from, the morning dose of ombitasvir/paritaprevir/ritonavir plus dasabuvir resulted in higher paritaprevir AUC (87\%-117\%) and is not recommended (Viekira Pak, 2017).

Elbasvir and grazoprevir were evaluated for interactions with ATV/r, DRV/r, and LPV/r. Due to a significantly higher observed grazoprevir AUC (650\%-1186\%) that may increase the risk of ALT elevations, use of these boosted protease inhibitors is contraindicated (Zepatier, 2018).

The impact of several boosted protease inhibitors on glecaprevir/ pibrentasvir pharmacokinetics was evaluated. Coadministration of glecaprevir/pibrentasvir with ATV plus ritonavir resulted in higher glecaprevir AUC $(\geq 553 \%)$ and $C_{\max }(\geq 306 \%)$ and higher pibrentasvir AUC $(\geq 64 \%)$ and $C_{\max }(\geq 29 \%)$; the coadministration of glecaprevir/ 
pibrentasvir with ATV plus ritonavir is contraindicated. Coadministration with DRV plus ritonavir resulted in higher glecaprevir AUC (397\%) and $C_{\max }(209 \%)$ with no change in pibrentasvir, whereas coadministration with LPV plus ritonavir resulted in higher glecaprevir AUC $(338 \%)$ and $C_{\max }(155 \%)$ and higher pibrentasavir AUC (146\%) and $C_{\max }(40 \%)$. Coadministration of glecaprevir/pibrentasvir with either DRV plus ritonavir or LPV plus ritonavir is not recommended.

\section{Integrase Inhibitors}

Integrase inhibitor-based regimens containing dolutegravir (DTG), EVG, or raltegravir (RAL) are potent third agents in HIV regimens, are well tolerated, and have low DDI potential (https://aidsinfo.nih.gov/ guidelines/html/1/adult-and-adolescent-arv/11/what-to-start). In vitro, DTG inhibits organic cation transporter OCT2, multidrug and toxin extrusion protein MATE1, and organic anion transporters OAT1 and OAT3 and is a UGT1A1 and CYP3A substrate (Tivicay, 2017). In vitro, EVG is a modest CYP2C9 inducer and a CYP3A substrate (Stribild, 2017). RAL is a UGT1A1 substrate with low propensity to affect other compounds (Isentress, 2015). Bictegravir inhibits OCT2 and MATE1 and is a substrate of UGT1A1 and CYP3A (Biktarvy, 2018).

Administration of simeprevir with RAL resulted in no interactions (Ouwerkerk-Mahadevan et al., 2012a). Administration of ledipasvir/ sofosbuvir with EVG (as EVG/COBI/FTC/TDF) resulted in no interaction with EVG; although higher ledipasvir (78\%), sofosbuvir $(36 \%)$, and GS-331007 (44\%) exposures were observed, all were attributable to COBI and none warranted dose adjustment (Garrison et al., 2015). Administration of ledipasvir/sofosbuvir with RAL or DTG resulted in no interactions (Kirby et al., 2012; German et al., 2014c; Garrison et al., 2015). No clinically significant interactions were observed between sofosbuvir/velpatasvir and RAL, DTG, or EVG from EVG/COBI/FTC/ TAF or EVG/COBI/FTC/TDF (Mogalian et al., 2016c, 2018). Similarly, no clinically significant interactions were observed between sofosbuvir/ velpatasvir/voxilaprevir and bictegravir/FTC/TAF or EVG/COBI/FTC/ TAF, supporting coadministration (Vosevi, 2017a; Biktarvy, 2018).

Administration of daclatasvir with DTG resulted in 33\% higher DTG exposure, with no change in daclatasvir exposure (Song et al., 2015).

Administration of ombitasvir/paritaprevir/ritonavir plus dasabuvir with RAL or DTG resulted in a higher RAL (134\%) or DTG $(38 \%)$ AUC due to UGT1A1 inhibition by ombitasvir, paritaprevir, and dasabuvir and/or CYP3A inhibition by ritonavir (Khatri et al., 2014b, 2016). No RAL or DTG dose adjustment is required (Viekira Pak, 2017).

No clinically significant interactions were observed with elbasvir and grazoprevir administered with DTG or RAL. Use of EVG/COBI/FTC/ $\mathrm{TDF}$ is not recommended with elbasvir/grazoprevir due to the presence of COBI within the regimen.

Higher glecaprevir AUC (205\%) and $C_{\max }(150 \%)$ and higher pibrentasvir AUC (57\%) were observed after coadministration of glecaprevir/pibrentasvir with EVG/COBI/FTC/TAF; no dose adjustment is required for their coadministration. RAL AUC and $C_{\max }$ values were $47 \%$ and $34 \%$ higher, respectively, after coadministration with glecaprevir/pibrentasvir; no dose adjustment is required (Mavyret, 2017).

\section{Discussion}

Chronic HCV infection affects individuals worldwide and is a significant cause of premature death via liver cirrhosis, functional failure, or hepatocellular carcinoma. Historical HCV treatments were poorly tolerated and insufficiently efficacious. Recently developed anti$\mathrm{HCV}$ therapies have consisted of combinations of all-oral regimens that include at least two (e.g., elbasvir and grazoprevir, glecaprevir and pibrentasvir, or sofosbuvir with daclatasvir, simeprevir, ledipasvir, or velpatasvir;) and up to five drugs (e.g., ombitasvir/paritaprevir/ritonavir plus dasabuvir with or without ribavirin). Because $\mathrm{HCV}$-infected individuals may use multiple medications for various ailments, the consideration of DDIs is critical for selection of a safe and effective regimen to treat $\mathrm{HCV}$.

The complexity of DDIs with DAA regimens varies significantly but can be clinically managed with knowledge. Sofosbuvir is a substrate for efflux transporters but is not a perpetrator of transport- or metabolizing enzyme (e.g., P450, UGT)-mediated interactions. Generally, NS5A inhibitors perpetrate transport-mediated but not enzymatic interactions and are not subject to many interactions requiring dose adjustment or restricted use except for inducers of P450s or efflux transporters. Interactions with $\mathrm{HCV}$ protease inhibitors can be complex, because they are generally sensitive to OATP and/or CYP3A inhibition and can perpetrate both transport- and enzyme-mediated (e.g., CYP, UGT) interactions and can be amplified if ritonavir is used to support the DAA regimen.

In summary, the availability of multiple highly effective DAA regimens has resulted in significant progress in curing large numbers of patients living with chronic HCV infection. The potential for DDIs is an important consideration when identifying an optimal treatment regimen for individual patients.

\section{Acknowledgments}

We gratefully acknowledge Svetlana Markova for editorial contributions to this manuscript.

\section{Authorship Contributions}

Wrote or contributed to the writing of the manuscript: Garrison, German, Mogalian, Mathias.

\section{References}

Badri P, Dutta S, Coakley E, Cohen D, Ding B, Podsadecki T, Bernstein B, Awni W, and Menon R (2015) Pharmacokinetics and dose recommendations for cyclosporine and tacrolimus when coadministered with ABT-450, ombitasvir, and dasabuvir. Am J Transplant 15:1313-1322.

Bifano M, Adamczyk R, Hwang C, Kandoussi H, Marion A, and Bertz RJ (2013) Daclatasvir pharmacokinetics in healthy subjects: no clinically relevant drug-drug interaction with either cyclosporine or tacrolimus [Poster]. 64th Annual Meeting of the American Association for the Study of Liver Diseases; 2013 Nov 1-2; Washington, DC.

Bifano M, Hwang C, Oosterhuis B, Hartstra J, Tiessen RG, Velinova-Donga M, Kandoussi H, Sevinsky H, and Bertz R (2012) Assessment of HIV antiretroviral drug interactions with the HCV NS5A replication complex inhibitor daclatasvir demonstrates a PK profile which supports coadministration with tenofovir, efavirenz and atazanavir/r [Poster]. 19th Conference on Retroviruses and Opportunistic Infections; 2012 Mar 5-8; Seattle, WA.

Bifano M, Sevinsky H, Hwang C, Kandoussi H, Jiang H, Grasela D, and Bertz R (2014) Effect of the coadministration of daclatasvir on the pharmacokinetics of a combined oral contraceptive containing ethinyl estradiol and norgestimate. Antivir Ther 19:511-519.

Biktarvy. (2018) Biktarvy (bictegravir, emtricitabine, and tenofovir alafenamide) tablets, for oral use (USPI). Gilead Sciences Inc., Foster City, CA.

Daklinza. (2014) Daklinza (daclatasvir) tablets $60 \mathrm{mg}$ (Japan Prescribing Information). BristolMyers Squibb K.K., Tokyo.

Daklinza. (2017) Daklinza (daclatasvir) tablets, for oral use (USPI). Bristol-Myers Squibb Co., Princeton, NJ.

Daklinza. (2018) Daklinza $30 \mathrm{mg}$ film-coated tablets, daklinza $60 \mathrm{mg}$ film-coated tablets (SmPC). Bristol-Myers Squibb Pharmaceutical Ltd., Middlesex, UK.

Eley T, Gardiner DF, Persson A, He B, You X, Shah V, Sherman D, Kandoussi H, Sims KD, Pasquinelli C, et al. (2011) Evaluation of drug interaction of the HCV protease inhibitor asunaprevir (ASV; BMS-650032) at $200 \mathrm{mg}$ twice daily in metabolic cocktail and P-glycoprotein (P-gp) probe studies in healthy volunteers [Abstract 381]. 62nd Annual Meeting of the American Association for the Study of Liver Diseases; 2011 Nov 4-8; San Francisco, CA.

Eley T, You X, Wang R, Luo W-L, Huang S-P, Kandoussi H, Bertz RJ, and Bifano M (2014) Daclatasvir: overview of drug-drug interactions with antiretroviral agents and other common concomitant drugs [Poster]. HIV DART; 2014 Dec 9-12; Miami, FL.

Epclusa. (2017a) Epclusa $400 \mathrm{mg} / 100 \mathrm{mg}$ film coated tablets (SmPC). Gilead Sciences Ireland UC, County Cork, Ireland.

Epclusa. (2017b) Epclusa (sofosbuvir and velpatasvir) tablets, for oral use (USPI). Gilead Sciences Inc., Foster City, CA

Epivir. (2013) Epivir (lamivudine) tablets and oral solution (USPI). GlaxoSmithKline, Research Triangle Park, NC.

Garimella T, You X, Wang R, Huang SP, Kandoussi H, Bifano M, Bertz R, and Eley T (2016) A review of daclatasvir drug-drug interactions. Adv Ther 33:1867-1884.

Garrison KL, Custodio JM, Pang PS, Das M, Cheng F, Ma GJ, and Mathias A (2015) Drug interactions between anti-HCV antivirals ledipasvir/sofosbuvir and integrase strand transfer 
inhibitor-based regimens [Poster 71]. 16th International Workshop on Clinical Pharmacology of HIV and Hepatitis Therapy; 2015 May 26-28; Washington, DC.

Garrison KL, Wang Y, Brainard DM, Sajwani K, and Mathias A (2014) The effect of rifampin on the pharmacokinetics of sofosbuvir in healthy volunteers [Poster 992]. 65th Annual Meeting of the American Association for the Study of Liver Diseases: The Liver Meeting; 2014 Nov 7-11; Boston

German P, Garrison K, Pang PS, Stamm L, Ray A, Shen G, Buacharern M, and Mathias A (2015) Drug interactions between the anti-HCV regimen ledipasvir/sofosbuvir and ritonavir boosted protease inhibitors plus emtricitabine/tenofovir DF [Presentation 82]. 22nd Conference on Retroviruses and Opportunistic Infections; 2015 Feb 23-26; Seattle, WA

German P, Moorehead L, Pang P, Vimal M, and Mathias A (2014a) Lack of a clinically important pharmacokinetic interaction between sofosbuvir or ledipasvir and hormonal oral contraceptives norgestimate/ethinyl estradiol in HCV-uninfected female subjects. J Clin Pharmacol 54: 1290-1298.

German P, Pang PS, Fang L, Chung D, and Mathias A (2014b) Drug-drug interaction profile of the fixed-dose combination tablet ledipasvir/sofosbuvir [Presentation 1976]. 65th Annual Meeting of the American Association for the Study of Liver Diseases: The Liver Meeting; 2014 Nov 7-11; Boston.

German P, Yang J, West S, Chung D, and Mathias A (2014c) Drug interactions between directacting anti-HCV antivirals sofosbuvir and ledipasvir and HIV antiretrovirals [Presentation]. 15th International Workshop on Clinical Pharmacology of HIV and Hepatitis Therapy; 2014 May 19-21; Washington, DC

Graham CS, Baden LR, Yu E, Mrus JM, Carnie J, Heeren T, and Koziel MJ (2001) Influence of human immunodeficiency virus infection on the course of hepatitis $\mathrm{C}$ virus infection: a metaanalysis. Clin Infect Dis 33:562-569.

Hammond GL, Abrams LS, Creasy GW, Natarajan J, Allen JG, and Siiteri PK (2003) Serum distribution of the major metabolites of norgestimate in relation to its pharmacological properties. Contraception 67:93-99.

Harvoni. (2017a) Harvoni 90 mg/400 mg film-coated tablets (SmPC). Gilead Sciences Ireland UC, County Cork, Ireland.

Harvoni. (2017b) Harvoni (ledipasvir and sofosbuvir) tablets, for oral use (USPI). Gilead Sciences Inc., Foster City, CA.

HCV Guidelines. (2015) HCV guidelines: recommendations for testing, managing, and treating hepatitis C. https://www.hcvguidelines.org/

Isentress. (2015) Isentress (raltegravir) film-coated tablets, for oral use; Isentress (raltegravir) chewable tablets, for oral use; Isentress (raltegravir) for oral suspension (USPI). Merck Sharp \& Dohme Corp., Whitehouse Station, NJ.

Kalliokoski A and Niemi M (2009) Impact of OATP transporters on pharmacokinetics. $\mathrm{Br}$ J Pharmacol 158:693-705.

Khatri A, Trinh R, Zhao W, Podsadecki T, and Menon R (2016) Drug-drug interaction between the direct-acting antiviral regimen of ombitasvir-paritaprevir-ritonavir plus dasabuvir and the HIV antiretroviral agent dolutegravir or abacavir plus lamivudine. Antimicrob Agents Chemother 60: $6244-6251$.

Khatri A, Wang T, Podsadecki T, Trinh R, Awni W, Dutta S, and Menon R (2014a) Drug-drug interactions of the direct acting antiviral regimen of ABT-450/r, ombitasvir and dasabuvi with emtricitabine + tenofovir, raltegravir, rilpivirine and efavirenz [Poster]. 54th Interscience Conference on Antimicrobial Agents and Chemotherapy; 2015 Sep 5-9; Washington, DC.

Khatri A, Wang T, Wang H, Podsadecki T, Trinh R, Awni W, Dutta S, and Menon R (2014b) Drug-drug interactions of the direct acting antiviral regimen of ABT-450/r, ombitasvir and dasabuvir with HIV protease inhibitors [Poster]. 54th Interscience Conference on Antimicrobial Agents and Chemotherapy; 2015 Sep 5-9; Washington, DC.

Kim WR, Lake JR, Smith JM, Skeans MA, Schladt DP, Edwards EB, Harper AM, Wainright JL, Snyder JJ, Israni AK, et al. (2017) OPTN/SRTR 2015 annual data report: liver. Am J Transplant 17 (Suppl 1):174-251.

Kirby B, Mathias A, Rossi S, Moyer C, Shen G, and Kearney BP (2012) No clinically significant pharmacokinetic interactions between sofosbuvir (GS-7977) and HIV antiretrovirals atripla, rilpivirine, darunavir/ritonavir, or raltegravir in healthy volunteers [Abstract P-1629]. 63rd Annual Meeting and Postgraduate Course of the American Association for the Study of Liver Diseases; 2012 Nov 9-13; Boston.

Kirby B, Mathias A, Yang C, Pang P, Moorehead L, Chung D, and Kearney BP (2013) Metabolism and excretion of ledipasvir (GS-5885) in humans [Poster O_22]. 8th International Workshop on Clinical Pharmacology of Hepatitis Therapy; 2013 Jun 26-27; Cambridge, MA.

Kirby BJ, Symonds WT, Kearney BP, and Mathias AA (2015) Pharmacokinetic, pharmacodynamic, and drug-interaction profile of the hepatitis C virus NS5B polymerase inhibitor sofosbuvir. Clin Pharmacokinet 54:677-690.

Kirby BJ, Taylor J, Stamm LM, Song Q, Wei H, Li Y, Alhelawe D, Ling KHJ, and Mathias A (2016a) Evaluation of transporter and cytochrome P450-mediated drug-drug interactions with the pan genotypic HCV NS3/4A protease inhibitor voxilaprevir (GS-9857) or sofosbuvir/ velpatasvir/voxilaprevir and phenotypic probe drugs [Presentation O24 and O25]. 17th International Workshop on Clinical Pharmacology of HIV and Hepatitis Therapy; 2016 Jun 8-10; Washington, DC

Kirby BJ, Taylor J, Stamm LM, Wei H, Hernandez M, Ling KHJ, and Mathias A (2016b) Metabolism and excretion of voxilaprevir (GS-9857), a pangenotypic HCV NS3/4A protease inhibitor in humans [Poster 57]. 17th International Workshop on Clinical Pharmacology of HIV and Hepatitis Therapy; 2016 Jun 8-10; Washington, DC

Krishna R, Bergman A, Larson P, Cote J, Lasseter K, Dilzer S, Wang A, Zeng W, Chen L, Wagner J, et al. (2007) Effect of a single cyclosporine dose on the single-dose pharmacokinetics of sitagliptin (MK-0431), a dipeptidyl peptidase-4 inhibitor, in healthy male subjects. J Clin Pharmacol 47:165-174.

Mahajan R, Xing J, Liu SJ, Ly KN, Moorman AC, Rupp L, Xu F, and Holmberg SD; Chronic Hepatitis Cohort Study (CHeCS) Investigators (2014) Mortality among persons in care with hepatitis C virus infection: the Chronic Hepatitis Cohort Study (CHeCS), 2006-2010. Clin Infect Dis 58:1055-1061.

Marshall WL, Feng HP, Caro L, Talaty J, Guo Z, Huang X, Panebianco D, Ma J, Mangin E, O'Reilly TE, et al. (2017) No clinically meaningful pharmacokinetic interaction between the hepatitis $\mathrm{C}$ virus inhibitors elbasvir and grazoprevir and the oral contraceptives ethinyl estradiol and levonorgestrel. Eur J Clin Pharmacol 73:593-600.

Mathias A, Cornpropst M, Clemons D, Denning J, and Symonds WT (2012) No clinically significant pharmacokinetic drug-drug interactions between sofosbuvir (GS-7977) and the immunosuppressants, cyclosporine A or tacrolimus in healthy volunteers. Hepatology 56: 1063-1064A.

Maviret. (2018) Maviret $100 \mathrm{mg} / 40 \mathrm{mg}$ film-coated tablets (SmPC). Abbvie Ltd., Berkshire, United Kingdom

Mavyret. (2017) Mavyret (glecaprevir and pibrentasvir) tablets, for oral use (USPI). AbbVie Inc., North Chicago, IL.

Menon RM, Badri PS, Wang T, Polepally AR, Zha J, Khatri A, Wang H, Hu B, Coakley EP, Podsadecki TJ, et al. (2015) Drug-drug interaction profile of the all-oral anti-hepatitis $\mathrm{C}$ virus regimen of paritaprevir/ritonavir, ombitasvir, and dasabuvir. J Hepatol 63:20-29.

Mogalian E, Brainard D, McNally J, Shen G, Cuvin J, and Mathias A (2014) Lack of clinically relevant pharmacokinetic drug-drug interaction between norgestimate/ethinyl estradiol and pangenotypic HCV NS5A inhibitor GS-5816 in HCV-uninfected female subjects [Poster 1998]. 65th Annual Meeting of the American Association for the Study of Liver Diseases: The Liver Meeting; 2014 Nov 7-11; Boston.

Mogalian E, Daryani V, Osinusi A, Mcnally J, Ni L, Wyles D, Brau N, Sulkowski M, Ling J, and Mathias A (2016a) Pharmacokinetics of sofosbuvir/velpatasvir and tenofovir in subjects with $\mathrm{HCV} / \mathrm{HIV}$ coinfection using boosted or unboosted antiretroviral regimens [Poster]. 67th Annual Meeting of the American Association for the Study of Liver Diseases: The Liver Meeting; 2016 Nov 11-15; Boston.

Mogalian E, German P, Kearney BP, Yang CY, Brainard D, McNally J, Moorehead L, and Mathias A (2016b) Use of multiple probes to assess transporter- and cytochrome P450-mediated drugdrug interaction potential of the pangenotypic HCV NS5A inhibitor velpatasvir. Clin Pharmacokinet 55:605-613.

Mogalian E, Stamm L, Osinusi A, Shen G, Sajwani K, McNally J, Ling J, and Mathias A (2015a) Drug-drug interaction studies between hepatitis $\mathrm{C}$ virus antivirals sofosbuvir and velpatasvir and HIV antiretroviral therapies [Poster 2265]. 66th Annual Meeting of the American Association for the Study of Liver Diseases; 2015 Nov 13-17; San Francisco, CA.

Mogalian E, Stamm L, Osinusi A, Shen G, Sajwani K, McNally J, Ling J, and Mathias A (2016c) Drug interaction studies between sofosbuvir/velpatasvir and boosted HIV ARV regimens [Presentation]. 23rd Conference on Retroviruses and Opportunistic Infections; 2016 Feb 22-25; Boston.

Mogalian E, Stamm LM, Osinusi A, Brainard DM, Shen G, Ling KHJ, and Mathias A (2018) Drug-drug interactions studies between HCV antivirals sofosbuvir/velpatasvir and boosted and unboosted HIV antiretroviral regimens in healthy volunteers. Clin Infect Dis DOI: 10.1093/cid/ ciy201 [published ahead of print].

Mogalian E, Yang C, Stamm LM, Shen G, Hernandez M, and Mathias A (2015b) Metabolism and excretion of pangenotypic HCV NS5A inhibitor GS-5816 in humans [Poster 72]. 16th International Workshop on Clinical Pharmacology of HIV and Hepatitis Therapy; 2015 May 2628; Washington, DC

Neuvonen PJ, Niemi M, and Backman JT (2006) Drug interactions with lipid-lowering drugs: mechanisms and clinical relevance. Clin Pharmacol Ther 80:565-581.

Norvir. (2017) Norvir $100 \mathrm{mg}$ film-coated tablets (SmPC). AbbVie Ltd., Berkshire, UK.

Neoral. (2009) Neoral soft gelatin capsules (cyclosporine capsules, USP) modified, Neoral ora solution (cyclosporine oral solution, USP) modified (USPI). Novartis Pharmaceuticals Corporation, East Hanover, $\mathrm{NJ}$

Olysio. (2016) Simeprevir 150 mg (SmPC). Janssen-Cilag AG, Zug, Switzerland.

Olysio. (2017) Olysio (simeprevir) capsules, for oral use (USPI). Janssen Therapeutics Division of Janssen Products, Titusville, NJ.

Ouwerkerk-Mahadevan S, Sekar V, Peeters M, and Beumont-Mauviel M (2012a) The pharmacokinetic interactions of $\mathrm{HCV}$ protease inhibitor TMC435 with rilpivirine, tenofovir, efavirenz or raltegravir in healthy volunteers [Presentation]. 19th Conference on Retroviruses and Opportunistic Infections; 2012 Mar 5-8; Seattle, WA.

Ouwerkerk-Mahadevan S, Sekar V, Simion A, Peeters M, and Beumont-Mauviel M (2012b) The pharmacokinetic interactions of the HCV protease inhibitor simeprevir (TMC435) with HIV antiretroviral agents in healthy volunteers [Poster]. IDWeek; 2012 Oct 17-21; San Diego, CA.

Ouwerkerk-Mahadevan S, Simion A, Mortier S, Peeters M, and Beumont-Mauviel M (2012c) No clinically significant interaction between the investigational $\mathrm{HCV}$ protease inhibitor simeprevir (TMC435) and the immunosuppressive agents cyclosporine and tacrolimus [Presentation]. 63rd Annual Meeting of the American Association for the Study of Liver Diseases; 2012 Nov 9-13, Boston.

Ouwerkerk-Mahadevan S, Simion A, Spittaels K, Peeters M, and Beumont-Mauviel M (2012d) No pharmacokinetic interaction between the investigational HCV protease inhibitor simeprevir (TMC435) and an oral contraceptive containing ethinylestradiol and norethindrone [Poster]. 63rd Annual Meeting of the American Association for the Study of Liver Diseases; 2012 Nov 9-13; Boston.

Ouwerkerk-Mahadevan S, Snoeys J, Peeters M, Beumont-Mauviel M, and Simion A (2016) Drugdrug interactions with the NS3/4A protease inhibitor simeprevir. Clin Pharmacokinet 55 : 197-208.

Prograf. (2003) Prograf tacrolimus capsules; tacrolimus injection (for intravenous infusion only) (USPI). Fujisawa Healthcare Inc., Deerfield, IL.

Reyataz. (2017) Reyataz (atazanavir) capsules, for oral use, Reyataz (atazanavir) oral powder (USPI). Bristol-Myers Squibb Co., Princeton, NJ.

Sekar V, Verloes R, Meyvisch P, Spittaels K, Akuma SH, and De Smedt G (2010) Evaluation of metabolic interactions for TMC435 via cytochrome P450 (CYP) enzymes in healthy volunteers [Poster 1076]. 45th Annual Meeting of the European Association for the Study of the Liver; 2010 Apr 14-18; Vienna, Austria.

Smith BD, Morgan ML, Beckett GA, Falck-Ytter Y, Holtzman D, Teo CG, Jewett A, Baack B, Rein DB, Patel N, et al.; Centers for Disease Control and Prevention (2012) Recommendation for the identification of chronic hepatitis C virus infection among persons born during 19451965. MMWR Recomm Rep 61:1-32.

Song I, Jerva F, Zong J, Choukour M, Wynne B, Buchanan A, Eley T, Huang S-P, and Ross L (2015) Evaluation of drug interactions between dolutegravir and daclatasvir in healthy subjects [Poster]. 16th International Workshop on Clinical Pharmacology of HIV and Hepatitis Therapy; 2015 May 26-28; Washington, DC.

Soriano V, Vispo E, Labarga P, Medrano J, and Barreiro P (2010) Viral hepatitis and HIV co-infection. Antiviral Res 85:303-315

Sovaldi. (2017a) Sovaldi (sofosbuvir) tablets, for oral use (USPI). Gilead Sciences Inc., Foster City, CA.

Sovaldi. (2017b) Sovaldi (sofosbuvir) $400 \mathrm{mg}$ film-coated tablets (SmPC). Gilead Sciences Ireland UC, County Cork Ireland. 
Stribild. (2017) Stribild $150 \mathrm{mg} / 150 \mathrm{mg} / 200 \mathrm{mg} / 245 \mathrm{mg}$ film-coated tablets (SmPC). Gilead Sciences Ltd., London, United Kingdom.

Suraweera D, Sundaram V, and Saab S (2016) Treatment of hepatitis C virus infection in liver transplant recipients. Gastroenterol Hepatol (N Y) 12:23-30.

Sustiva. (2017) Sustiva (efavirenz) capsules for oral use, Sustiva (efavirenz) tablets for oral use (USPI). Bristol-Myers Squibb Co., Princeton, NJ.

Technivie. (2016) Technivie (ombitasvir, paritaprevir and ritonavir) tablets, for oral use (USPI) AbbVie Inc., North Chicago, IL.

Tivicay. (2017) Tivicay (dolutegravir) tablets, for oral use (USPI). GlaxoSmithKline, Research Triangle Park, NC.

Tong L, Phan TK, Robinson KL, Babusis D, Strab R, Bhoopathy S, Hidalgo IJ, Rhodes GR, and Ray AS (2007) Effects of human immunodeficiency virus protease inhibitors on the intestinal absorption of tenofovir disoproxil fumarate in vitro. Antimicrob Agents Chemother $\mathbf{5 1}$ $3498-3504$.

Truvada. (2017) Truvada (emtricitabine/tenofovir disoproxil fumarate) tablets, for oral use (USPI) Gilead Sciences Inc., Foster City, CA.

Viekira Pak. (2017) Viekira Pak (ombitasvir, paritaprevir, and ritonavir tablets; dasabuvir tablets), co-packaged for oral use (USPI). AbbVie Inc., North Chicago, IL.
Viekirax. (2018) Viekirax $12.5 \mathrm{mg} / 75 \mathrm{mg} / 50 \mathrm{mg}$ film-coated tablets (SmPC). Abbvie Ltd., Berkshire, UK.

Vosevi. (2017a) Vosevi (sofosbuvir, velpatasvir, and voxilaprevir) tablets, for oral use (USPI) Gilead Sciences Inc., Foster City, CA

Vosevi. (2017b) Vosevi $400 \mathrm{mg} / 100 \mathrm{mg} / 100 \mathrm{mg}$ film-coated tablets sofosbuvir/velpatasvir/ voxilaprevir (SmPC). Gilead Sciences Ireland UC, County Cork, Ireland.

Zepatier. (2017) Zepatier $50 \mathrm{mg} / 100 \mathrm{mg}$ film coated tablets (SmPC). Merck Sharp \& Dohme Ltd., Hertfordshire, UK.

Zepatier. (2018) Zepatier (elbasvir and grazoprevir) tablets, for oral use (USPI). Merck \& Co. Inc., Whitehouse Station, NJ.

Zhang H, Cui D, Wang B, Han YH, Balimane P, Yang Z, Sinz M, and Rodrigues AD (2007) Pharmacokinetic drug interactions involving 17alpha-ethinylestradiol: a new look at an old drug. Clin Pharmacokinet 46:133-157.

Address correspondence to: Kimberly L. Garrison, Gilead Sciences Inc., 333 Lakeside Dr., Foster City, CA 94404. E-mail: kim.garrison@gilead.com 